NBER WORKING PAPER SERIES

\title{
PREVENTIVES VERSUS TREATMENTS REDUX: TIGHTER BOUNDS ON DISTORTIONS IN INNOVATION INCENTIVES WITH AN APPLICATION TO THE GLOBAL DEMAND FOR HIV PHARMACEUTICALS
}

\author{
Michael Kremer \\ Christopher M. Snyder \\ Working Paper 24206 \\ http://www.nber.org/papers/w24206
NATIONAL BUREAU OF ECONOMIC RESEARCH
1050 Massachusetts Avenue
Cambridge, MA 02138
January 2018

The authors are grateful to the editor, Victor Tremblay, and anonymous reviewers for suggestions that substantially improved the paper; to Margaret Kyle, Paul Novosad, Douglas Staiger, Robert Staiger, Heidi Williams, and seminar participants at Penn State for insightful comments; and to Matthew Goodkin-Gold and Henry Senkfor for excellent research assistance. Henry Senkfor's assistantship was funded by Dartmouth College's Presidential Scholars program, which the authors gratefully acknowledge. The views expressed herein are those of the authors and do not necessarily reflect the views of the National Bureau of Economic Research.

NBER working papers are circulated for discussion and comment purposes. They have not been peerreviewed or been subject to the review by the NBER Board of Directors that accompanies official NBER publications.

(C) 2018 by Michael Kremer and Christopher M. Snyder. All rights reserved. Short sections of text, not to exceed two paragraphs, may be quoted without explicit permission provided that full credit, including (c) notice, is given to the source. 
Preventives Versus Treatments Redux: Tighter Bounds on Distortions in Innovation Incentives with an Application to the Global Demand for HIV Pharmaceuticals

Michael Kremer and Christopher M. Snyder

NBER Working Paper No. 24206

January 2018

JEL No. F23,I14,L65,O31

\begin{abstract}
$\underline{\text { ABSTRACT }}$
Kremer and Snyder (2015) show that demand curves for a preventive and treatment may have different shapes though they target the same disease, biasing the pharmaceutical manufacturer toward developing the lucrative rather than the socially desirable product. This paper tightens the theoretical bounds on the potential deadweight loss from such biases. Using a calibration of the global demand for HIV pharmaceuticals, we demonstrate the dramatically sharper analysis achievable with the new bounds, allowing us to pinpoint potential deadweight loss at $62 \%$ of the global gain from curing HIV. We use the calibration to perform policy counterfactuals, assessing welfare effects of government policies such as a subsidy, reference pricing, and price-discrimination ban. The fit of our calibration is good: we find that a hypothetical drug monopolist would price an HIV drug so high that only $4 \%$ of the infected population worldwide would purchase, matching actual drug prices and quantities in the early 2000 s before subsidies in low-income countries ramped up.
\end{abstract}

Michael Kremer

Harvard University

Department of Economics

Littauer Center M20

Cambridge, MA 02138

and NBER

mkremer@fas.harvard.edu

Christopher M. Snyder

Department of Economics

Dartmouth College

301 Rockefeller Hall

Hanover, NH 03755

and NBER

chris.snyder@dartmouth.edu

A data appendix is available at http://www.nber.org/data-appendix/w24206 


\title{
Preventives Versus Treatments Redux: Tighter Bounds on Distortions in Innovation Incentives with an Application to the Global Demand for HIV Pharmaceuticals
}

\author{
Michael Kremer \\ Harvard University \\ Center for Global Development \\ National Bureau of Economic Research
}

\author{
Christopher M. Snyder \\ Dartmouth College \\ National Bureau of Economic Research
}

November 2017

\begin{abstract}
Kremer and Snyder (2015) show that demand curves for a preventive and treatment may have different shapes though they target the same disease, biasing the pharmaceutical manufacturer toward developing the lucrative rather than the socially desirable product. This paper tightens the theoretical bounds on the potential deadweight loss from such biases. Using a calibration of the global demand for HIV pharmaceuticals, we demonstrate the dramatically sharper analysis achievable with the new bounds, allowing us to pinpoint potential deadweight loss at $62 \%$ of the global gain from curing HIV. We use the calibration to perform policy counterfactuals, assessing welfare effects of government policies such as a subsidy, reference pricing, and price-discrimination ban. The fit of our calibration is good: we find that a hypothetical drug monopolist would price an HIV drug so high that only $4 \%$ of the infected population worldwide would purchase, matching actual drug prices and quantities in the early 2000s before subsidies in low-income countries ramped up
\end{abstract}

JEL codes: O31 (Innovation and Invention: Processes and Incentives), L65 (Industry Studies: Manufacturing: Drugs), F23 (International Business), I14 (Health and Inequality)

Keywords: pharmaceuticals, deadweight loss, product development

Contact information: Michael Kremer: Department of Economics, Harvard University, Littauer Center 207, Cambridge MA 02138; email: mkremer@fas.harvard.edu. Christopher Snyder: Department of Economics, Dartmouth College, 301 Rockefeller Hall, Hanover NH 03755; email: chris.snyder@dartmouth.edu.

Acknowledgments: The authors are grateful to the editor, Victor Tremblay, and anonymous reviewers for suggestions that substantially improved the paper; to Margaret Kyle, Paul Novosad, Douglas Staiger, Robert Staiger, Heidi Williams, and seminar participants at Penn State for insightful comments; and to Matthew Goodkin-Gold and Henry Senkfor for excellent research assistance. Henry Senkfor's assistantship was funded by Dartmouth College's Presidential Scholars program, which the authors gratefully acknowledge. 


\section{Introduction}

In previous work (Kremer and Snyder, 2015), we argued that a drug may be more lucrative than a vaccine, even when both products target the same disease and thus have the same health benefit, even in the absence of epidemiological externalities. The argument is that a vaccine is sold before consumers contract the disease, when consumers differ considerably in disease risk, preventing the firm from easily extracting much of their surplus with a uniform price. On the other hand, a drug is sold after consumers have contracted the disease, when they no longer differ in disease risk, allowing the manufacturer to extract more surplus from these relatively homogeneous consumers. The drug may thus end up being more lucrative and the manufacturer biased toward developing it over the vaccine even if the vaccine is substantially more effective or lower cost. ${ }^{1}$ We calibrated the distribution of infection risk in the U.S. population for human immunodeficiency virus (HIV) and found it to be close to Zipf, i.e., a power-law distribution with exponent equal to 1. A Zipf distribution of disease risk intuitively means that each doubling of risk cuts the number of consumers with at least that risk in half, leading to an iso-revenue property. In view of our theoretical result that, owing to this iso-revenue property, the Zipf risk distribution generates the worst bias against preventives, our previous work provided one explanation for why a variety of HIV drugs have been developed but as yet no vaccine.

The present paper contributes along both empirical and theoretical dimensions. The main contribution is empirical. We expand the calibrations of demand for HIV pharmaceuticals beyond the U.S. market, calibrating a global demand curve using country-level data on disease prevalence, factoring in the joint distribution of income using data on per capita gross domestic product (GDP) and considering a range of values of the income elasticity of healthcare expenditures for robustness. For consistency, throughout most of the analysis we interpret calibrated world demand in a similar way as Kremer and Snyder (2015) did U.S. demand, i.e., reflecting the individual purchase decisions on a (perhaps counterfactual) private market in the absence of intervention by governments or insurance companies and in the absence of epidemiological externalities. ${ }^{2}$ How-

\footnotetext{
${ }^{1}$ Kremer and Snyder (2015) show that this bias may be reversed when income (or more generally willingness to pay) covaries sufficiently negatively with disease risk (see their Proposition 18). The bias against vaccines described in the footnoted paragraph arises in a setting with little or no income variation (covered by their Proposition 3 ) or in a setting with independent income and disease-risk distributions (covered by their Proposition 16).

${ }^{2}$ For theoretical analyses of vaccine markets in the presence of epidemiological externalities, see Brito, Sheshinski, and Intrilligator (1991); Francis (1997); Geoffard and Philipson (1997); Gersovitz (2003); Gersovitz and Hammer
} 
ever, the calibrations can be interpreted more broadly. We offer an alternative interpretation of world demand as reflecting purchases by national agencies on behalf of citizens in their health systems. This interpretation allows for consumer heterogeneity and epidemiological externalities within countries.

Using the calibrated global demand for an HIV drug, we show that-in the absence of international price discrimination, subsidies in low-income countries, or regulatory threats to intellectual property - a profit-maximizing monopolist would charge such a high drug price that only about $4 \%$ of infected individuals worldwide would buy the treatment. These estimates are remarkably close to the actual prices and quantities of antiretroviral therapies (ARTs) in 2003, the year we focus on in the subsequent analysis as arguably approximating the "state of nature" before subsidies or other policy interventions became widespread. We estimate that the deadweight loss from charging such a high price for an existing product (the area of the Harberger (1954) triangle) amounts to about half of the global benefit from curing HIV.

The resulting calibrated demands are employed to examine a range of policy issues. One exercise leverages Kremer and Snyder's (2015) formulas for worst-case bounds on deadweight loss from pharmaceutical sales on the private market. Since the greatest conceivable distortions turn out to occur at the extensive margin (whether a product is developed at all) rather than the intensive margin (how high the mark up is for an existing product, generating a Harberger deadweightloss triangle), the formulas hinge on the percentage of the surplus that would be generated by completely relieving the disease burden that the producer can extract for itself (we refer to this as the producer-surplus ratio, denoted $\rho$ ). The producer-surplus ratio in turn depends on how the demand curve is shaped. Using the calibrated demands, we can compute $\rho$ for a monopoly producer of either a vaccine $(v)$ or a drug $(d)$. In our baseline calibration (indicated by superscript 0 ), we obtain a producer-surplus ratio of $\rho_{v}^{0}=44 \%$ for an HIV vaccine. Considering the market for an HIV vaccine in isolation, this leads to a worst-case bound on deadweight loss of $100-44=56 \%$. In other words, the distorted incentives provided by the private market to the firm regarding the vaccine's development and price could dissipate as much as $56 \%$ of the global benefit from curing HIV. Moving to the isolated drug market, our baseline estimate of the drug-producer-surplus ratio is $\rho_{d}^{0}=38 \%$, leading to a worst-case bound on deadweight loss of $100-38=62 \%$.

(2004, 2005); Chen and Toxvaerd (2014); as well as our own work (Kremer, Snyder, and Williams 2012). 
Rather than consider the vaccine and drug markets in isolation, it is natural to wonder what the worst-case deadweight loss is in the comprehensive case in which either or both products can be produced. The theoretical result relevant to this comprehensive case is Proposition 15 of Kremer and Snyder (2015). We have been able to tighten the bounds, as reported in Proposition 3 of this paper. For canonical values of model parameters, these tighter bounds lead to an exact expression for worst-case deadweight loss as reported in Proposition 2. The exact expression simply equals the larger of the two worst-case bounds for the isolated products; i.e., using the numbers from the calibration, $\max (56,62)=62 \%$. The previous result merely told us that worst-case deadweight in the comprehensive case is no less than the difference between the worst cases for the individual products, i.e., no less than $62-56=6 \%$. The new bound thus represents a dramatic sharpening of the analysis in this application: instead of saying that worst-case deadweight loss lies somewhere in the interval $[6 \%, 100 \%]$, we can now pinpoint it at $62 \%$.

The fit of our demand calibration appears to be good. Based on the calibrated global demand for an HIV drug, we calculate that-in the absence of international price discrimination, subsidies in low-income countries, or regulatory threats to intellectual property-a profit-maximizing monopolist would charge such a high drug price that only about $4 \%$ of infected individuals worldwide would buy the treatment. These price and quantity estimates are remarkably close to actual price and quantity of antiretroviral therapies (ARTs) in 2003, the baseline year for our analysis, arguably approximating the "state of nature" before subsidies or other policy interventions became widespread. The deadweight loss associated with this high price (the area of the Harberger (1954) triangle) amounts to nearly half of the global benefit from curing HIV.

Since the calibrations require assumptions about particular parameter values, we gauge the robustness of the results by providing calibrations for a range of values of these parameters. The key parameter turns out to be the income elasticity of healthcare expenditure, $\epsilon$. We first consider the baseline case of unit income elasticity, which happens to be close to leading estimates using international data (e.g., Newhouse (1977) estimates $\epsilon=1.3$ ). We provide calibrations for a wide range of $\epsilon$ spanning these values as well as $\epsilon=0$, which is equivalent to a model in which consumers vary only in disease risk. Because the disease-risk distribution is even more Zipf similar than the distribution of the product of risk and income, the potential for deadweight loss is enormous in the model with only disease risk, for example, reaching $70 \%$ of the overall disease burden in the 
vaccine market.

We also use the calibrations to perform a variety of counterfactual exercises, assessing the welfare gains and losses from government interventions such as a subsidy, reference pricing, and price-discrimination ban. Regarding a subsidy, owing to the Zipf-similar shape of demand in the baseline vaccine calibration, a small subsidy is enough to swing the equilibrium from a high price at which few consumers are served to universal vaccination. Thus universal vaccination may be a more robust public policy than previously thought, possible to rationalize even in the absence of epidemiological externalities, and possible to effectuate without a mandate. Regarding reference pricing, supposing all other countries peg their prices to some proportion, $u$, of the United States', a monopoly manufacturer of either a vaccine or a drug would prefer to serve the United States even if the ceiling were as low as $u=0.2$, i.e., $20 \%$ of the U.S. price. This result highlights the pivotal U.S. role in the pharmaceutical market, explaining why other countries may be emboldened to free ride. Regarding price discrimination, cross-country price discrimination can be quite lucrative in our model because each country's consumers are homogeneous, so one different price per country is all that is needed to accomplish perfect price discrimination and attain the social optimum. Hence, banning price discrimination can be quite distortionary, leading to a $56 \%$ decline in vaccine producer surplus and a $62 \%$ decline in drug producer surplus, declines equal to the potential increase in deadweight loss in these markets.

As discussed, the most closely related paper in the past literature is Kremer and Snyder (2015). ${ }^{3}$ Other of our past related work includes Kremer, Snyder, and Williams (2012), which focuses on epidemiological externalities, and Kremer and Snyder (2016), which generalizes the bounds on deadweight loss to product markets beyond pharmaceuticals. ${ }^{4}$

A detailed discussion of the connection between this line of our research and other authors' work is provided in Kremer and Snyder (2015). Here we highlight just two key connections. The present paper contributes to the literature on incentives for innovation in R\&D-intensive industries (see, e.g., Newell, Jaffee, and Stavins 1999; Acemoglu and Linn 2004; Finkelstein 2004; and

\footnotetext{
${ }^{3}$ Kremer and Snyder (2015) was initially circulated as a series of National Bureau of Economic Research working papers (Kremer and Snyder 2003, 2013). The international calibration provided in Section 6 of the 2013 working paper but cut from the 2015 published version became the germ of the present paper. Besides Figure 4, the other calibrations as well as all the theoretical results are new developments.

${ }^{4}$ Kremer and Snyder (2016) also include calibrations of international demand. Since they analyze general product markets, their calibrations include only income, not disease risk. Unlike the present calibrations, theirs accounts for within-country heterogeneity by allowing each country to have a different lognormal distribution of income.
} 
Budish, Roin, and Williams 2015). Most closely related are studies of innovation in healthcare markets by Lakdawalla and Sood (2013) and especially Garber, Jones, and Romer (2006). The latter paper relates static and dynamic deadweight loss to the shape of the demand curve as we do. They focus on a different distortion, that coinsurance can induce overconsumption and excess entry by defraying a fraction of the pharmaceutical price, whereas distortion in our model is due to underconsumption and too little entry. The present paper also contributes to the literature on the revenue and welfare consequences of government interventions in the international pharmaceutical market. See, e.g., Kyle (2007), Sood et al. (2008), and a series of papers by Patricia Danzon and coauthors, including Danzon and Ketcham (2005) and Danzon, Wang, and Wang (2005).

The paper is organized as follows. The next section presents the model. Section 3 provides the propositions tightening the bound on deadweight loss from our previous work. The rest of the paper focuses on the calibrations. Section 4 describes the methods and (5) the data used in the calibrations. Section 6 presents the results from calibrations for the baseline scenario and Section 7 for scenarios involving price discrimination. Section 8 presents calibrations for alternative parameterizations to explore robustness. Section 9 concludes. Proofs of propositions are provided in the appendix.

\section{Model}

We base our analysis on the most general model in Kremer and Snyder (2015). This model, appearing in their Section $\mathrm{V}$, is general in several respects. First, it allows for general values of the parameters $c_{j}, s_{j}$, and $e_{j}$ defined below. Second, it allows for multiple sources of consumer heterogeneity embodied in the random variables $X \in[0,1]$, representing disease risk, $Y \geq 0$, representing willingness to pay to avoid a unit of disease harm (for simplicity, this can be thought of as income or wealth), and $H \geq 0$, representing the consumer's draw of disease harm conditional on contracting it, where $H$ has unit mean and is mean-independent of $X$ and $Y$, i.e., $E(H)=E(H \mid Y=y)=E(H \mid X=x)=1$. Ex ante, the consumer draws realizations $x$ and $y$ of random variables $X$ and $Y$. Ex post, the consumer's disease status is a draw from a Bernoulli distribution with probability $x$ of contracting it. Conditional on contracting the disease, the consumer draws a realization $h$ of harm $H$. Letting $B$ denote total disease burden from an ex ante perspective 
and $N$ be the mass of consumers, we have $B=N E(X Y H)=N E(X Y)$, where the last equality follows from the assumptions on $H$. Proposition 14 in Kremer and Snyder (2015) shows that the three random variables $X, Y$, and $H$ are sufficient to capture quite general models of consumer heterogeneity.

A monopoly pharmaceutical manufacturer can develop two products indexed by $j$ to sell on the private market to consumers: a vaccine $(j=v)$ and/or a drug $(j=d)$. If the firm chooses to develop product $j$, it must pay fixed cost $k_{j} \geq 0$, reflecting expenditures on research, capacity, etc. Let $c_{j} \geq 0$ denote product $j$ 's constant marginal production cost and $s_{j} \geq 0$ the harm from its side effects. Let $e_{j} \in[0,1]$ denote its efficacy or conversely $r_{j}=1-e_{j}$ denote its failure rate. To simplify the notation, we ignore discounting (or take all values to reflect ex ante present discounted values).

For consistency with Kremer and Snyder (2015), throughout most of the discussion we maintain the interpretation of demand as reflecting the individual purchase decisions on a private market in the absence of government intervention, insurance, or epidemiological externalities. ${ }^{5}$ There is an alternative interpretation of demand that allows these factors to be integrated into the analysis. Rather than assuming consumers are homogeneous within each country, one can assume national governments (or large national insurers) decide whether to order a pharmaceutical on behalf of heterogeneous citizens enrolled their health systems. This interpretation handles epidemiological externalities under the assumption that they mostly flow within country borders and would be internalized by the national agent. We return to a discussion of this alternative interpretation in the conclusion, mentioning caveats required for it to apply.

The key distortion in the model is not a government interventions, externality, or consumer behavioral bias but rather the problem of surplus appropriability. The key difference between the products in the model is that a vaccine is purchased before the consumer has contracted the disease and so still faces an uncertain disease risk; a drug is purchased after disease risk has been resolved into binary disease status (infected or not). This difference can generate different shapes for the

\footnotetext{
${ }^{5}$ We do not deny the importance of epidemiological externalities_-indeed some of our other work (Kremer, Snyder, and Williams 2010) focuses exclusively on such externalities-but want to focus on other distortions in this paper. Epidemiological externalities can be shut down as a source of distortion by assuming that the pharmaceuticals, while preventing individuals from experiencing disease symptoms, do not slow transmission of an infectious disease. An alternative way to shut down epidemiological externalities would be to consider non-infectious conditions such as heart attacks. The alternative interpretation of demand reflecting purchases by a national agent discussed next can incorporate some forms of epidemiological externality.
} 
pharmaceuticals' demand curves, leading to differences between the producer's ability to appropriate surplus with the two products. It is well known since Arrow (1962) that the monopolist's inability to appropriate consumer surplus and Harberger deadweight loss results in suboptimal innovation incentives. An additional distortion here is created by the producer's bias toward the more lucrative product. Obviously, the producer will prefer the product with lower values of the R\&D cost $\left(k_{j}\right)$, marginal production cost $\left(c_{j}\right)$, side effects $\left(s_{j}\right)$, and ineffectiveness $\left(r_{j}\right)$, but we do not call this preference a bias because it is shared by the social planner: both agree low values are better. Our analysis focuses on the wedge between private and social innovation incentives, arising here due to factors affecting surplus appropriability. Maximizing the wedge between private and social innovation incentives will not be as simple as maximizing the difference between, say, $k_{v}$ and $k_{d}$.

We proceed with the specification of the model by introducing notation for the firm's decisions. Letting $\sigma$ denote the firm's product-development strategy, it has four possibilities: producing the vaccine alone $(\sigma=v)$, the drug alone $(\sigma=d)$, both products $(\sigma=b)$, or neither product $(\sigma=n)$. Consider the continuation game following its having developed exactly one of the products, i.e., $\sigma=j$ for $j \in\{v, d\}$. Let $p_{j}$ denote price and $Q_{j}\left(p_{j}\right)$ the demand curve for product $j$. (Demand is also a function of $s_{j}$ and $r_{j}$, but arguments besides $p_{j}$ are suppressed in the notation for brevity.) Let $P S_{j}\left(p_{j}\right)=\left(p_{j}-c_{j}\right) Q_{j}\left(p_{j}\right)$ denote producer surplus, $C S_{j}\left(p_{j}\right)=\int_{p_{j}}^{\infty} Q_{j}(x) d x$ consumer surplus, and $T S_{j}\left(p_{j}\right)=P S_{j}\left(p_{j}\right)+C S_{j}\left(p_{j}\right)$ total surplus. Note $P S_{j}$ and $T S_{j}$ are surpluses from an ex post perspective-i.e., treating $k_{j}$ as a sunk cost and thus ignoring it. Profit from an ex ante perspective-i.e., treating $k_{j}$ as an economic cost—is denoted $\Pi_{j}\left(p_{j}\right)=P S_{j}\left(p_{j}\right)-k_{j}$. Ex ante social welfare is denoted $W_{j}\left(p_{j}\right)=T S_{j}\left(p_{j}\right)-k_{j}$.

Next consider the continuation game following the firm's having developed both products, i.e., $\sigma=b$. Let $p_{b v}$ denote the price it sets for the vaccine and $p_{b d}$ for the drug. With both products available, the demand for vaccines $Q_{b v}\left(p_{b}\right)$ and for drugs $Q_{b d}\left(p_{b}\right)$ in general are functions of the vector of both prices, $p_{b}=\left(p_{b v}, p_{b d}\right)$. These demands can be tied back to the single-product demand curves: $Q_{j}\left(p_{j}\right)=Q_{b j}\left(p_{j}, \infty\right)$. Denote the vector-valued demand function when both products are produced as $Q_{b}\left(p_{b}\right)=\left(Q_{b v}\left(p_{b}\right), Q_{b d}\left(p_{b}\right)\right)$. Letting $c_{b}=\left(c_{v}, c_{d}\right)$, we can write producer surplus from both products as the dot product $P S_{b}\left(p_{b}\right)=\left(p_{b}-c_{b}\right)^{\prime} Q_{b}\left(p_{b}\right)$. Consumer surplus is

$$
C S_{b}\left(p_{b}\right)=\int_{p_{b v}}^{\infty} Q_{b v}\left(p_{v}, p_{b d}\right) d p_{v}+\int_{p_{b d}}^{\infty} Q_{b d}\left(p_{d}, p_{b v}\right) d p_{d}
$$


and total surplus is $T S_{b}\left(p_{b}\right)=C S_{b}\left(p_{b}\right)+P S_{b}\left(p_{b}\right)$.

Next consider the continuation game following the firm's developing no product, i.e., $\sigma=n$. Nothing can be sold if nothing has been developed. Thus, for all $p_{n} \geq 0, Q_{n}\left(p_{n}\right)=0$, implying

$$
P S_{n}\left(p_{n}\right)=C S_{n}\left(p_{n}\right)=T S_{n}\left(p_{n}\right)=0 \text {. }
$$

Equilibrium values are denoted with a single star. For example, $p_{\sigma}^{*}=\operatorname{argmax}_{p} P S_{\sigma}(p) \operatorname{denotes}$ the monopoly price, $q_{\sigma}^{*}=Q_{\sigma}\left(p_{\sigma}^{*}\right)$ monopoly quantity, $P S_{\sigma}^{*}=P S_{\sigma}\left(p_{\sigma}^{*}\right)$ monopoly producer surplus, $W_{\sigma}^{*}$ denote monopoly welfare, etc. (Note that $p_{\sigma}^{*}$ and $q_{\sigma}^{*}$ are vectors if $\sigma=b$.) Rather than an arbitrary product strategy $\sigma$, we will often be interested in equilibrium values associated with the equilibrium product strategy $\sigma^{*}$. To streamline notation, we denote these values by starring the relevant variable but dropping the product-strategy subscript: i.e., $p^{*}=p_{\sigma^{*}}^{*}, q^{*}=q_{\sigma^{*}}^{*} P S^{*}=P S_{\sigma^{*}}^{*}$, $W^{*}=W_{\sigma^{*}}^{*}$, etc. Variables with two stars denote socially optimal values. For example, $p_{\sigma}^{* *}$ denotes the socially optimal price. We have $p_{j}^{* *}=c_{j}$ if a single product $j$ is produced and $p_{b}^{* *}=\left(c_{v}, c_{d}\right)$, the vector of marginal costs, if both are produced. Furthermore, for any product strategy $\sigma$, we have $P S_{\sigma}^{* *}=0$ and $T S_{\sigma}^{* *}=C S_{\sigma}^{* *}$. Rather than an arbitrary product strategy, $\sigma$, we will often be interested in socially optimal values under the socially optimal product strategy, $\sigma^{* *}$. To streamline notation, we denote these values by double starring the relevant variable but dropping the product-strategy subscript: i.e., $p^{* *}=p_{\sigma^{* *}}^{* *}, q^{* *}=q_{\sigma^{* *}}^{* *}, P S^{* *}=P S_{\sigma^{* *}}^{* *}, W^{* *}=W_{\sigma^{* *}}^{* *}$, etc.

While the analysis allows for general values of the parameters $c_{j}, s_{j}$, and $r_{j}$, the values $c_{j}=s_{j}=$ $r_{j}=0$, which we refer to as benchmark values, play a special role, allowing us to "level the playing field" for the two products in all respects save for the timing of when they are sold. ${ }^{6}$ To denote equilibrium under benchmark parameters, we replace the star with a zero in the superscript. For example, $p^{0}$ denotes the monopoly price under the equilibrium product strategy for benchmark parameters, and $P S^{0}$ denotes the resulting equilibrium producer surplus. To denote the social optimum under benchmark parameters, we replace the two stars with two zeros in the superscript. For example, $p^{00}$ denotes the socially optimal price under the socially optimal product strategy for

\footnotetext{
${ }^{6}$ For example, allowing for positive values of $c_{v}$ and $c_{d}$, the normalization $c_{v}=c_{d}$ equalizes the cost of producing a dose but introduces a bias in the aggregate cost of a universal pharmaceutical program. In particular, universal vaccination would be more costly than universal drug treatment by a factor equal to the reciprocal of the prevalence rate. In addition, the benchmark parameters are associated with the most extreme worst-case bounds under some conditions; see Proposition 12 from Kremer and Snyder (2015).
} 
benchmark parameters, and $W^{00}$ denotes the resulting social welfare in this social optimum.

Suppose that the firm chooses a non-trivial product strategy $\sigma \in\{v, d, b\}$ under benchmark parameters. Since products are costless to manufacture, the socially optimal pricing policy is to give the products away: $p_{\sigma}^{00}=0$. Since products have no side effects, all consumers purchase; and since products are perfectly effective, their consumption relieves the entire disease burden. Therefore,

$$
T S_{\sigma}^{00}=B \quad \text { for } \sigma \in\{v, d, b\}
$$

\section{Bounding Deadweight Loss}

The central results of this section are a series of propositions providing new bounds on deadweight loss. Before presenting the propositions, we define several deadweight loss concepts that we work with.

\subsection{Harberger Deadweight Loss}

We refer to the area of Harberger's (1954) triangle, equal to the deadweight loss stemming from prices above marginal cost, as Harberger deadweight loss, denoted $H D W L_{\sigma}^{*}$, where $\sigma$ indexes the product strategy under consideration. We have $H D W L_{\sigma}^{*}=T S_{\sigma}^{* *}-T S_{\sigma}^{*}=T S_{\sigma}^{* *}-P S_{\sigma}^{*}-C S_{\sigma}^{*}$. Deadweight loss from all sources including distortions due to a possible mismatch between the product strategy $\sigma$ and the socially efficient one, $\sigma^{* *}$, is denoted $D W L_{\sigma}^{*}=W^{* *}-W_{\sigma}^{*}$. As before, we can condition on the firm's equilibrium product strategy $\sigma^{*}$ rather than arbitrary product strategy $\sigma$, writing $H D W L^{*}=T S_{\sigma^{*}}^{* *}-T S^{*}$ and $D W L^{*}=W^{* *}-W^{*}$.

It will be useful to work with relative versions of these deadweight loss measures, normalizing by disease burden as a measure of the size of the market. Thus, relative Harberger and total deadweight losses are $H D W L_{\sigma}^{*} / B$ and $D W L_{\sigma}^{*} / B$, respectively, when conditioned on an arbitrary product strategy $\sigma$; and $H D W L^{*} / B$ and $D W L^{*} / B$, respectively, when conditioned on the equilibrium product strategy $\sigma^{*}$.

The analysis proceeds by trying to find simple characterizations of these relative deadweight

\footnotetext{
${ }^{7}$ Tirole (1988) proposes slightly different expressions for relative deadweight loss, dividing by first-best social surplus rather than disease burden. By equation (2), our relative concepts coincide with his for benchmark parameters.
} 
loss concepts. Substituting for $H D W L_{\sigma}^{*}$, we have

$$
\frac{H D W L_{\sigma}^{*}}{B}=\frac{T S_{\sigma}^{* *}}{B}-\frac{P S_{\sigma}^{*}}{B}-\frac{C S_{\sigma}^{*}}{B}=\frac{T S_{\sigma}^{* *}}{B}-\rho_{\sigma}^{*}-\gamma_{\sigma}^{*}
$$

where $\rho_{\sigma}^{*}=P S_{\sigma}^{*} / B$ is relative producer surplus and $\gamma_{\sigma}^{*}=C S_{\sigma}^{*} / B$ is relative consumer surplus. Allowing the firm to pursue the equilibrium product strategy $\sigma^{*}$, it follows from equation (3) that

$$
\frac{H D W L^{*}}{B}=\frac{T S_{\sigma^{*}}^{* *}}{B}-\rho^{*}-\gamma^{*}
$$

where relative surpluses are defined in the obvious way: $\rho^{*}=P S^{*} / B$ and $\gamma^{*}=C S^{*} / B$. Assuming benchmark parameter values and further that the equilibrium product strategy is non-trivial (i.e., $\sigma^{0} \in\{v, d, b\}$ ), equation (2) implies

$$
\frac{H D W L^{0}}{B}=1-\rho^{0}-\gamma^{0}
$$

\subsection{Comprehensive Deadweight Loss}

No such simple formulas are available for the more comprehensive measure of relative deadweight loss, $D W L^{*} / B$, which involves both pricing and product-choice distortions. The approach of Kremer and Snyder (2015) was to focus instead on finding a simple formula for the worst case - the supremum - on this relative deadweight loss. Though they did not find an exact expression for the supremum, they found a lower bound on it, reported in their Proposition 15.

In this subsection, we provide a series of propositions that tighten that bound. The series of propositions apply to increasingly rich environments. The first proposition assumes benchmark parameters and considers the market for one product $j$ in isolation. The second proposition allows for multiple products to be developed. The third proposition generalizes the parameters beyond benchmark values. ${ }^{8}$

\footnotetext{
${ }^{8}$ To trace out the precise connection between the series of propositions provided here and our past results, Proposition 1 here superficially resembles Proposition 2 of Kremer and Snyder (2015) but they are subtly different. The previous result applied to the case in which both products could be produced but there is heterogeneity in $X$ alone. Proposition 1 here allows for heterogeneity in both $X$ and $Y$ but assumes only product $j$ can be produced. In fact, Proposition 1 here is a corollary of Theorem 1 of Kremer and Snyder (2016) for the special case of benchmark parameters. The translation of that result into the present context is somewhat involved, so instead we provide a direct proof here. Propositions 2 and 3 are new results. The assumptions behind Proposition 3 are identical to those behind Propo-
} 
Under benchmark parameters, we can derive exact expressions for the supremum on relative deadweight loss. The first proposition restricts attention to the market for a single product $j$ in isolation, putting aside the possibility of developing the other product alone or both. The worst case for deadweight loss arises when $k_{j}$ exceeds $P S_{j}^{0}$. The firm develops nothing in equilibrium, so the whole of first-best social welfare constitutes deadweight loss. In the limit as $k_{j}$ approaches $P S_{j}^{0}$ from above, deadweight loss approaches $T S_{j}^{00}-P S_{j}^{0}=B-P S_{j}^{0}$. Dividing by $B$ to express in relative terms gives the expression for the supremum in the next proposition. A formal proof is provided in the appendix.

Proposition 1. Suppose that only product $j=v$ or $j=d$ can be developed, not both. Fix the parameters at their benchmark values $c_{j}=s_{j}=r_{j}=0$. The supremum on relative deadweight loss is exactly $1-\rho_{j}^{0}$ :

$$
\sup _{\left\{k_{j} \geq 0\right\}}\left(\frac{D W L^{0}}{B}\right)=1-\rho_{j}^{0} .
$$

The next proposition allows for the development of a second product, requiring some additional notation to state. Let $P S_{\min }^{*}=\min \left\{P S_{v}^{*}, P S_{d}^{*}\right\}$ and $P S_{\max }^{*}=\max \left\{P S_{v}^{*}, P S_{d}^{*}\right\}$, respectively, be the minimum and maximum producer surpluses from the two individual products; and let $\rho_{\min }^{*}=$ $P S_{\min }^{*} / B$ and $\rho_{\max }^{*}=P S_{\max }^{*} / B$ be the corresponding relative measures. Let $P S_{\min }^{0}, P S_{\max }^{0}, \rho_{\min }^{0}$, and $\rho_{\max }^{0}$ be the corresponding variables in equilibrium under benchmark parameters.

The proof, provided in the appendix, establishes that the greatest distortion again arises when nothing is produced because development costs are too high. The whole of first-best social welfare again constitutes deadweight loss. The supremum on deadweight loss is approached in the limit as the development cost in the market for the less lucrative product approaches producer surplus in that market from above (keeping the development cost above producer surplus in the other market). Suppose for concreteness that the vaccine is the less lucrative of the two products. Then the supremum on deadweight loss is approached in the limit $k_{v} \downarrow P S_{\min }^{0}$ for $k_{d}$ sufficiently high (taking the limit $k_{d} \uparrow \infty$ suffices). Expressed relative to $B$, this supremum equals $1-\rho_{v}^{0}$. More generally, the supremum is associated with whichever of the two isolated product markets is less lucrative. In such a market, a low development cost can generate a large equilibrium distortion without dissipating too much first-best social welfare, leaving the greatest potential for deadweight loss.

sition 15 of Kremer and Snyder (2015), so the results are directly comparable. Proposition 3 tightens the previous bound. 
The supremum on deadweight loss when there are two products equals the greater of the suprema in equation (6) for the two isolated markets: $\max \left(1-\rho_{v}^{0}, 1-\rho_{d}^{0}\right)=1-\min \left(\rho_{v}^{0}, \rho_{d}^{0}\right)=1-\rho_{\min }^{0}$.

Proposition 2. Consider a model of the pharmaceutical market with multiple sources of consumer heterogeneity, still fixing the parameters at their benchmark values $c_{j}=s_{j}=r_{j}=0$ but allowing the firm to produce either or both of the products. The supremum on relative deadweight loss is exactly $1-\rho_{\min }^{0}$ :

$$
\sup _{\left\{k_{v}, k_{d} \geq 0\right\}}\left(\frac{D W L^{0}}{B}\right)=1-\rho_{\min }^{0} .
$$

The final proposition generalizes the parameters beyond benchmark values. The proof is immediate from the previous proposition. The supremum restricting parameters to take benchmark values is weakly lower than that allowing those parameters to be free. Thus the supremum for benchmark parameters from the previous proposition is a lower bound on the supremum over general parameters in the next proposition.

Proposition 3. Consider a model of the pharmaceutical market with multiple sources of consumer heterogeneity and general values of the parameters $c_{j}, s_{j}, r_{j} \geq 0$. The supremum on relative deadweight loss is at least $1-\rho_{\min }^{0}$ :

$$
\sup _{\left\{k_{j}, c_{j}, s_{j}, r_{j} \geq 0 \mid j=v, d\right\}}\left(\frac{D W L^{*}}{B}\right) \geq 1-\rho_{\min }^{0} .
$$

This bound is directly comparable to the bound we derived for the case of multiple products and general parameters in our earlier work, Kremer and Snyder (2015) Proposition 15. The earlier result stated that the supremum on relative deadweight loss is no less than $\rho_{\max }^{0}-\rho_{\min }^{0}$. We derived that bound by focusing on just one possible distortion, that arising from the firm's developing the "wrong" product, the less socially efficient but more lucrative one. The supremum on that distortion equals $\rho_{\max }^{0}-\rho_{\min }^{0}$ in relative terms. The new bound focuses on a potentially larger source of deadweight loss. Society stands to lose more when the firm develops not just a different product but nothing in place of the socially efficient product. The new bound $1-\rho_{\min }^{0}$ reflects the larger gap between no product and the efficient one.

It is obvious that the new bound is weakly tighter since $\rho_{\max }^{0} \leq 1$ implies $1-\rho_{\min }^{0} \geq \rho_{\max }^{0}-\rho_{\min }^{0}$. To understand how much tighter it can be in practice, consider a scenario in which neither the vaccine nor the drug is particularly lucrative, i.e., $\rho_{v}^{0} \approx \rho_{d}^{0} \approx 0$. Using the old bound, $\rho_{\max }^{0}-\rho_{\min }^{0} \approx 0$, we arrive at the tautological conclusion that the supremum on deadweight must be positive. The 
new bound, $1-\rho_{\min }^{0} \approx 1$, raises the real prospect that nearly the whole disease burden can be dissipated in this scenario. Just such a scenario will play out in the calibrations.

\subsection{Zipf Worst Case}

The analysis so far asked what fixed costs and other parameters generate the worst deadweight loss for a given demand curve. Kremer and Snyder (2015) proceed to ask what demand curve shapes generate the worst deadweight loss among those with the same area underneath (the same $B$ in our setting). The answer is what they term the symmetrically truncated Zipf (STRZ) demand. A Zipf distribution is the special case of a power-law distribution with an exponent equal to 1, which in the disease context intuitively means that each doubling of disease risk cuts the number of consumers with at least that risk in half. The resulting demand curve is unit elastic, implying that all feasible prices generate the same revenue, and hence the same producer surplus under benchmark parameters. Put another way, no price is especially lucrative with this demand curve; all generate the same (low) producer surplus. Example STRZ demands are provided later in Figures 4 and 6; they can be visualized as rectangular hyperbolas truncated at the top and bottom. The truncations are technical features keeping both the highest conceivable consumer value and the area under the curve constant.

Kremer and Snyder (2015) proposed a measure of how close a demand curve comes to the STRZ worst case, called the Zipf similarity of demand. Kremer and Snyder (2016) extended this result, providing the necessary adjustments to apply this formula to general settings with arbitrary scaling of the price and quantity axes. Adapting the formula from equation (16) of Kremer and Snyder (2016) to reflect the present notation, the Zipf similarity of the demand for pharmaceutical $j$ under benchmark parameters, $Z_{j}^{0}$, is

$$
Z_{j}^{0}=\frac{1-\rho_{j}^{0}}{1-\underline{\rho}\left(\mu_{j}^{0}\right)},
$$

where $\underline{\rho}\left(\mu_{j}^{0}\right)$ is the producer-surplus ratio of the associated STRZ demand. By Proposition 1, Zipf similarity is simply the supremum on deadweight loss for pharmaceutical $j$ relative to the supremum on deadweight loss for the associated STRZ demand. If the supremum on deadweight loss for pharmaceutical $j$ is $50 \%$ that of the STRZ worst case, then we say the demand for pharma- 
ceutical $j$ is $50 \%$ Zipf similar. To have a supremum on deadweight loss close to the STRZ worst case, all points on the demand curve for pharmaceutical $j$ must lie in a neighborhood around the STRZ demand curve. We will use equation (9) to measure the Zipf similarity of calibrated demand curves in Section 6.

To fill in some technical details behind equation (9), by the "associated" STRZ demand curve, we mean the STRZ demand in the same class as the demand for pharmaceutical $j$, where the class is indexed by the so-called mean-to-peak ratio, $\mu_{j}^{0}$, equal to the ratio of the mean consumer value for product $j$ in the social optimum to the highest value in the population. ${ }^{9}$ By Proposition 3 of Kremer and Snyder (2016), the producer-surplus ratio for this STRZ demand curve equals

$$
\underline{\rho}\left(\mu_{j}^{0}\right)=\frac{-1}{L W_{-1}\left(-\mu_{j}^{0} / e\right)},
$$

where $L W$ is the Lambert $\mathrm{W}$ function, the inverse relation $W(z)$ of the function $z=W e^{W}$. The -1 subscript on $L W$ denotes the lower branch of this relation. ${ }^{10}$

\section{Calibration Methodology}

This section describes the methods used to calibrate the new bounds on potential deadweight loss in the market for HIV pharmaceuticals. ${ }^{11}$ There are a number of advantages to assuming benchmark values of the parameters: $c_{j}=s_{j}=r_{j}=0$ for $j=v, d$. In addition to those discussed in footnote 6 , another advantage is that for benchmark parameters Proposition 2 gives an exact expression for

\footnotetext{
${ }^{9}$ Adapting the formula for from Lemma 1 of Kremer and Snyder (2015) to the present context, for the vaccine market we have

$$
\mu_{v}^{0}=\frac{\sum_{i=1}^{I} N_{i} X_{i} Y_{i}}{N(X Y)_{(I)}}
$$

where $N=\sum_{i=1}^{I} N_{i}$ is total population size and $(X Y)_{(I)}$ denotes the maximum order statistic for the product of $X_{i}$ and $Y_{i}$, i.e., the maximum value that $X_{i} Y_{i}$ takes on across countries. For the drug market,$$
\mu_{d}^{0}=\frac{\sum_{i=1}^{I} N_{i} X_{i} Y_{i}}{Y_{(I)} \sum_{i=1}^{I} N_{i} X_{i}},
$$

where $Y_{(I)}$ denotes the maximum value of $Y_{i}$ across countries.

${ }^{10}$ The branches of $L W$ are built-in functions in standard mathematical software packages including Mathematica, Matlab, and R. Other ways to compute $\underline{\rho}\left(\mu_{j}^{0}\right)$ besides equation (10) include reading the value from the graph in Kremer and Snyder's (2015) Figure 4 or taking the value from the tabulation in Kremer and Snyder's (2016) Table 2.

${ }^{11}$ We call this a "calibration" rather than an "estimation" exercise because we assume convenient forms for demand and cost and we fix certain important parameters (including $c_{j}, s_{j}, r_{j}$, and the income elasticity) rather than estimating them from price and quantity data.
} 
the supremum on deadweight loss rather than a bound. We thus maintain benchmark parameters throughout the remainder of the analysis. Following our earlier notation, we use a zero superscript (instead of a star) to indicate equilibrium values evaluated at the benchmark parameters and two zeros in the superscript (rather than two stars) to indicate socially optimal values under benchmark parameters.

By Proposition 2, calibration of the supremum on deadweight loss under benchmark parameters reduces to calibration of $\rho_{\min }^{0}$, which by the definition $\rho_{\min }^{0}=\min \left(\rho_{v}^{0}, \rho_{d}^{0}\right)$ in turn reduces to calibration of demand for a vaccine alone and a drug alone. We are exempted from having to calibrate the more complicated demands that apply when both products are offered because they do not show up in the formula. To calibrate demand for a vaccine alone and drug alone, we need to return to the random variables $X, Y$, and $H$ introduced at the outset of Section 2 and specify their distributions in the consumer market. We take the consumer market to consist of the entire world population. Country $i \in\{1, \ldots, I\}$ has a population of $N_{i}>0$ risk-neutral consumers. Consumers within a country are homogeneous, each having the same disease risk $X_{i} \in(0,1]$ and income $Y_{i}>0$. We have no reason to believe that the medical consequences of HIV infection differ across countries - indeed, untreated HIV is fatal everywhere. Thus, we abstract from cross-country variation in $H_{i}$, adopting the normalization $H_{i}=1$. We defer to the conclusion a discussion of a reinterpretation of the model allowing for heterogeneous consumers within a country assuming the national government makes purchases on their behalf.

As an intermediate step in deriving pharmaceutical demands, consider the counterfactual situation of a consumer who surely becomes infected. Assume that the elasticity of an individual's healthcare demand with respect to income is a constant $\epsilon$ across income and across individuals. For this to be the case, his willingness to pay to avoid harm from the disease as a function of his income $Y_{i}$ must take the form $Y_{i}^{\epsilon} \cdot{ }^{12}$ Now move from the counterfactual case in which the consumer

\footnotetext{
${ }^{12}$ The most general form preserving the property of constant income elasticity is $A_{i} Y_{i}^{\epsilon}$, allowing the leading coefficient to vary across consumers by taking it to be a random variable. We do not allow for that source of heterogeneity because doing so would introduce a third random variable characterizing consumers in a country, contradicting the maintained assumption that consumers are fully characterized by just $X_{i}$ and $Y_{i}$. A form that is more general but does not introduce a third source of heterogeneity is $A Y_{i}^{\epsilon}$, with a leading coefficient $A$ that is constant across consumers. Our specification of willingness to pay normalizes $A=1$. For most of our analysis, this normalization is without loss of generality since all of our surplus calculations will be expressed as a proportion of disease burden; $A$ is a scale factor which divides out of the proportion. In our analysis of prices, which is done in levels, the combined normalizations $A=1$ and $H_{i}=1$ comport with World Health Organization procurement thresholds as discussed below.
} 
is surely infected to the factual case in which he has disease risk $X_{i}$. His expected benefit from a vaccine is the product of this risk and the benefit of avoiding sure harm, i.e., $X_{i} Y_{i}^{\epsilon}$. If the firm's product strategy is to produce the vaccine alone, global vaccine demand is

$$
Q_{v}\left(p_{v}\right)=\sum_{i=1}^{I} \mathbf{1}\left(X_{i} Y_{i}^{\epsilon} \geq p_{v}\right) N_{i}
$$

where $\mathbf{1}(\cdot)$ is the indicator function, equal to 1 if the statement in parentheses is true and 0 otherwise. Turning to demand for a drug, because it is sold ex post, after disease status has been realized, any consumer who has contracted the disease buys a drug sold at price $p_{d}$ as long as $Y_{i}^{\epsilon} \geq p_{d}$. In expectation, $N_{i} X_{i}$ consumers in country $i$ end up contracting the disease. Thus global drug demand is

$$
Q_{d}\left(p_{d}\right)=\sum_{i=1}^{I} \mathbf{1}\left(Y_{i}^{\epsilon} \geq p_{d}\right) N_{i} X_{i}
$$

Demands when the firm produces both products are more complicated. As mentioned above, the supremum on deadweight loss can be calibrated without having to solve for the $\sigma^{0}=b$ continuation equilibrium. We report these demands for completeness but relegate them to a footnote. ${ }^{13}$

Equations (11) and (12) provide the foundation for our calibrations. To translate these formulas into demand units, the only additional elements required are country-level data on $N_{i}, X_{i}$, and $Y_{i}$ (described in the next section) and an assumption about the income elasticity, $\epsilon$. The practical implementation details are best discussed in the context of a simple example. Consider the toy example in Table 1 of a world with two countries. We first show how (11) can be used calibrate vaccine demand. Setting $\epsilon$ to the value we take for our baseline scenario, $\epsilon=1$, and maintaining benchmark parameters (no side effects and perfect efficacy), country $i$ 's willingness to pay for a vaccine equals the product $X_{i} Y_{i}$. As shown in the last column, country 1 has lower willingness to

\footnotetext{
${ }^{13}$ With benchmark values of the parameters $s_{j}=r_{j}=0$, one can show

$$
\begin{aligned}
& \tilde{Q}_{v}\left(p_{b v}, p_{b d}\right)=\sum_{i=1}^{I} \mathbf{1}\left(X_{i} \geq p_{v} / p_{d}\right) \mathbf{1}\left(X_{i} Y_{i}^{\epsilon} \geq p_{v}\right) N_{i} \\
& \tilde{Q}_{d}\left(p_{b d}, p_{b v}\right)=\sum_{i=1}^{I} \mathbf{1}\left(X_{i} \leq p_{v} / p_{d}\right) \mathbf{1}\left(Y_{i}^{\epsilon} \geq p_{d}\right) N_{i} X_{i} .
\end{aligned}
$$
}

With general values of $s_{j}$ and $r_{j}$, the expressions become considerably more complicated. Among other things, with imperfect efficacy, a consumer who purchases a vaccine than turns out to be ineffective may later purchase the drug as well. 
pay, $X_{1} Y_{1}=100$, than country 2 , at $X_{2} Y_{2}=400$. These willingness-to-pay numbers are the only relevant pricing points for a monopolist wishing to extract as much surplus as possible. According to equation (11), vaccine demand equals the cumulative population of countries having consumers with a willingness to pay at least as high as the given price: $Q_{v}(400)=N_{2}=1,500$ and $Q_{v}(100)=$ $N_{1}+N_{2}=2,500$. The resulting demand curve is the step function shown in Panel A of Figure 1. Under the benchmark assumption of costless production, vaccine producer surplus equals vaccine revenue, $P S_{v}(400)=400 \cdot 1,500=675,000$ and $P S_{v}(100)=100 \cdot 2,500=250,000$, implying $P S_{v}^{0}=675,000$, the area of the shaded rectangle in Panel A.

Next, we use (12) to calibrate drug demand. Willingness to pay for this product equals $Y_{i}$. The only two relevant pricing points for a monopolist are thus 10,000 and 9,000. Demand at these pricing points equals the cumulative infected population, $N_{i} X_{i}$, of countries with at least that willingness to pay: $Q_{d}(10,000)=N_{1} X_{1}=10$ and $Q_{d}(9,000)=N_{1} X_{1}+N_{2} X_{2}=85$. The resulting demand curve is shown in Panel B of Figure 1. Drug producer surplus is $P S_{d}(10,000)=100,000$ and $P S_{d}(9,000)=765,000$, implying $P S_{d}^{0}=765,000$, the area of the shaded rectangle in Panel B.

In this toy example, the drug is more lucrative than the vaccine. Intuitively, most of the heterogeneity across consumers in this toy example can be traced to $X_{i}$, which is five times higher in country 2 than 1 . This heterogeneity disappears by time the drug is sold because disease risk resolves into disease status, allowing the monopolist to extract almost all the available surplus$P S_{d}^{0}=765,000$ is $99 \%$ of the total disease burden, $B=775,000$-from drug consumers, who as Panel B of Figure 1 shows are virtually homogeneous. More formally, a comparison of demand formulas (11) and (12) reveals that they are identical except for the shifting of $X_{i}$ outside of the indicator function's argument in drug demand (12). This shift of course changes the shape of the demand curve as the move from Panel A to B in Figure 1 illustrates. Whether this shape change leads to a more or less lucrative demand curve in general is impossible to say-cases can be constructed in which $\rho_{v}^{0}>\rho_{d}^{0}$ and and other cases in which the reverse inequality holds-depending on the joint distribution of $N_{i}, X_{i}$, and $Y_{i}$. More concrete conclusions are available when there is little heterogeneity in $Y_{i}$. An immediate corollary of Kremer and Snyder's (2015) Proposition 3 is that if income is homogeneous across countries, i.e., if $Y_{i}=\bar{Y}$, and at least two countries have different positive values of $X_{i}$, then $\rho_{d}^{0}>\rho_{v}^{0}$. By continuity, this inequality still holds if $Y_{i}$ varies across countries as long as the variance is sufficiently small. This is the case in the toy example, in 
which $Y_{1}$ is only $11 \%$ higher than $Y_{2}$.

We use the same methods to calibrate demand with actual cross-country data as used here in the toy example. The demand curve will still be a step function but with finer steps due to the large number of actual countries. As in the toy example, the number of relevant pricing points for the monopolist is no greater than the number of countries. Only this manageable number of prices need to be checked to compute equilibrium (producer surplus maximizing) prices. Equilibrium prices and quantities can be combined with the calibrated demand curves to calculate surpluses, ratios of surpluses to disease burden, and the deadweight loss supremum.

\section{Data}

This section discusses the data sources for $N_{i}, X_{i}$, and $Y_{i}$ used in the calibrations. A preliminary question is what year would provide the best snapshot of the market for HIV pharmaceuticals. While the best year is the current one for most applications, this is not necessarily the case here. To calibrate demand for HIV pharmaceuticals, we would like some measure of the burden of the disease in the state of nature without any pharmaceuticals. Referring to the lower dotted curve in Figure 2, a growing percentage of people living with HIV have been receiving antiretroviral treatment (ART), from the negligible percentage in 2000 to nearly half in the most recent data. This expansion of treatment may have reduced transmission, and coupled with other initiatives to curtail the spread of HIV and other epidemiological trends resulted in a decline in HIV prevalence after its peak in 2003 (see the upper solid curve in Figure 2). The dip turned out to be short lived, rising again after 2010. This more recent rise may belie good news, reflecting a decline in HIV mortality resulting from the expansion of ART treatment. We select 2003 as our target year for data collection, a time when HIV prevalence reached a local peak while ART coverage was still fairly negligible.

The key inputs into our policy simulations are calibrations of the demand for a vaccine in (11) and a drug in (12). These equations contain three variables for which we need data: population $N_{i}$, disease risk $X_{i}$, and income $Y_{i}$ for countries $i=1, \ldots, I$. We obtained $N_{i}$ and $Y_{i}$ from the World Bank Open Data website, using gross domestic product (GDP) per capita for $Y_{i}$. We obtained $X_{i}$ from a UNAIDS publication (UNAIDS 2004a). ${ }^{14}$ Table 2 provides more details on the data sources and

\footnotetext{
${ }^{14}$ The UNAIDS website used as the source for the aggregate trends displayed in Figure 2 would be a natural source
} 
descriptive statistics for the main variables. Our dataset includes 158 countries. $^{15}$

Figure 3 maps the geographical distribution of $X_{i}$ and $Y_{i}$. The top panel shows the well-known concentration of HIV risk in sub-Saharan Africa. This would lead these countries to be the highest demanders of HIV pharmaceuticals in the calibration but for the fact, shown in the lower panel, that many are among the lowest income in the world, reducing their willingness to pay in the calibration. Table 2 reports a negative correlation between $X_{i}$ and $Y_{i}$ of -0.19 . Some countries such as the United States and South Africa are above the median in both $X_{i}$ and $Y_{i}$ and presumably will be centers of high demand for HIV pharmaceuticals in the calibration.

\section{Calibrations for Baseline Scenario}

This section presents calibrations for the baseline scenario in which countries are heterogeneous in both disease risk $X_{i}$ and income $Y_{i}$, the income elasticity is taken to be $\epsilon=1$, and the parameters are set at benchmark values $c_{j}=s_{j}=r_{j}=0$. Under these assumptions, a consumer in country $i$ has ex ante willingness to pay $X_{i} Y_{i}$ for a vaccine and ex post willingness to pay $Y_{i}$ for a drug conditional on being infected. Our analysis of the baseline calibration proceeds by first considering the vaccine market in isolation and then the drug market in isolation. We then combine these separate results to compute a comprehensive bound on deadweight loss using Proposition 2. We conclude the section with an analysis of whether and how equilibrium in the baseline calibration can be improved with government subsidies.

This baseline specification has some practical appeal beyond its use of round numbers. Interpret the normalization of harm $H_{i}=1$ as meaning that the pharmaceutical saves one Disability Adjusted Life Year (DALY). This is roughly the case for HIV drugs, as taking a year's course of ARTs roughly extends an infected person's life for that year. (Applying this interpretation to an HIV vaccine requires more care: we either have to assume that a yearly booster shot is needed or, if the vaccine provides more permanent protection, have to scale up the health benefit by the discounted flow of DALYs saved.) Under this interpretation, the baseline assumption that a con-

for country-level HIV data. However, the website seems to have expunged current and historical data for a substantial number of countries including the United States. We thus relied on a historical publication (UNAIDS 2004a) to recover country-level HIV data.

${ }^{15}$ Our data includes all countries with substantial populations except for Iraq, North Korea, Saudi Arabia, and Turkey, which are excluded because of missing HIV data. Other sources (UNAIDS 2004b) report very low prevalence rates for these countries, so their omission likely has little effect on our results. 
sumer is willing to pay annual per-capita GDP $Y_{i}$ to avoid $H_{i}$ is the same as saying that he or she is willing to spend a year of income to save a year of life. The standard of the World Health Organization (WHO) is that a highly cost effective health intervention saves a disability adjusted life year (DALY) at a cost less than the country's GDP per capita (see Hutubessy, et al., 2003). Thus our baseline assumption is simply that consumers purchase according to WHO standards.

As discussed, the calibrations can reinterpreted as applying to the purchase of pharmaceuticals by national governments on behalf of their citizens. This interpretation has the appeal of incorporating possible consumer heterogeneity and epidemiological externalities within countries. According to this interpretation, the baseline assumption is simply that WHO standards guide national purchases.

\subsection{Vaccine Market}

The top panel of Figure 4 shows the calibrated (inverse) demand curve for a vaccine alone. Following the methodology laid out in Section 4, it is constructed by ordering countries by the product $X_{i} Y_{i}$ and then sequentially plotting that value on the vertical axis after stepping off a quantity given the country's population $N_{i}$ on the horizontal axis.

Since we have assumed production is costless $\left(c_{v}=0\right)$, the price that maximizes producer surplus for the vaccine monopolist equivalently maximizes its revenue. Finding the price that maximizes producer surplus boils down to the geometric problem of finding the rectangle of largest area that can be inscribed underneath the demand curve. Holding the total area underneath the demand curve constant, some shapes are of course more conducive to inscribing large rectangles underneath than others. As discussed in Section 3.3, the worst shape is the symmetrically truncated Zipf (STRZ) demand, drawn in the figure as the grey curve. Notice that the calibrated demand curve largely overlaps the STRZ curve except where the United States appears, leading the demand curve to "belly out" there. The reason for the outsize influence of the United States on the market is that its consumers have very high incomes relative to most others, coupled with a not insubstantial HIV risk. These factors generate a large value of the product $X_{i} Y_{i}$ for the large population of U.S. consumers. U.S. consumers turn out to be the marginal ones in the calibration: the producersurplus-maximizing price just induces them to buy and strictly induces purchases by consumers in Botswana, South Africa, Swaziland, Bahamas, Namibia, Trinidad and Tobago, and Gabon. While 
consumers in these other countries are poorer than in the United States, their extremely high HIV prevalence rates result in their being higher demanders.

Further details of the calibration are provided in Table 4 . The vaccine sells at a price of $\$ 130$ to 344 million consumers. These numbers require some context, the price in particular since, as discussed in footnote 12 , it depends on an arbitrary constant scaling the willingness to pay function. The price at almost eight times the mean vaccine-consumer value is quite high, leading to a quantity that is quite low, less than $6 \%$ of the global population is served. All but 10 million of buyers live in South Africa and the United States. Producer surplus is $44 \%$ of the social surplus $B$ from completely relieving the disease burden. This $44 \%$ is the ratio of the shaded rectangle to the area under the inverse demand curve in the top panel of Figure 4 . Consumers obtain $16 \%$ of $B$. The residual, $41 \%$, is Harberger deadweight loss $H D W L_{v}^{0}$. The supremum on $D W L_{v}^{0}$ is achieved in the limit as $k_{v}$ approaches producer surplus-44\% of $B$-from above. The formula in Proposition 1 yields that this supremum equals $100-44=56 \%$ of $B$. A lack of adequate incentives to enter the market could dissipate more than half of the social surplus from completely relieving the disease burden.

Using the formula for the Zipf similarity, $Z_{j}^{0}$, of the demand for product $j$ provided by equation (9), we obtain $Z_{v}^{0}=0.66$, i.e., the calibrated vaccine demand curve is $66 \%$ similar to the STRZ worst case in that it generates $66 \%$ of the deadweight loss bound generated by the STRZ curve. This measure quantifies the moderately Zipf similar shape of the calibrated vaccine demand curve.

\subsection{Drug Market}

We next turn to the market for the drug alone. The calibrated (inverse) demand curve for a drug is shown in the lower panel of Figure 4. Given that a drug is only sold to consumers who contract the disease, it would not be surprising to see it sell at a much higher price to a much smaller group of consumers than a vaccine. The scale for drug price on the vertical axis is 100 times that for vaccine price, but the scale for drug quantity on the horizontal axis is only $1 / 100$ that for vaccine quantity. The combined scaling of the axes maintains the property that a unit of area reflects the same revenue and same surplus in both panels. Following the methodology laid out in Section 4, the drug demand curve is constructed by ordering countries in terms of $Y_{i}$ (i.e., GDP per capita), reflecting consumers' ex post willingness to pay for a drug conditional on contracting the disease. 
We then sequentially plot that value on the vertical axis after stepping off quantity $N_{i} X_{i}$, equal to the expected number of people in the country who contract the disease.

The producer-surplus-maximizing price is $\$ 27,400$. To provide some context for this price (which as explained in the previous subsection depends on an arbitrary scaling constant), it is extremely high, over ten times the mean of positive drug-consumer values. At this price, marginal consumers in Italy and inframarginal consumers in the 17 other higher-income countries (including the United States) purchase. Although these countries have a combined population of 783 million, only 1.4 million units end up being sold. These rich countries have a relatively low HIV prevalence rate (an average of $0.12 \%$ ), so relatively few people end up contracting the disease and needing an HIV drug. The 1.4 million consumers served represent a small fraction, less than $4 \%$, of the 38 million infected individuals in the world market for the drug.

The difference in the shape of the distribution of consumer values for a drug versus a vaccine leads the drug to be less lucrative than the vaccine. The drug producer obtains only $38 \%$ of the social surplus $B$ from completely relieving the disease burden, six percentage points less than with a vaccine, corresponding to the smaller area of the shaded producer-surplus rectangle in the lower compared to the upper panel. The shape of the drug demand curve is quite close to the STRZ worst case, drawn as a grey curve. Formally, the index of Zipf similarity is $Z_{d}^{0}=0.74$. Its shape is not conducive to inscribing a rectangle of substantial area underneath. Consumers obtain only $13 \%$ of $B$. The residual $49 \%$ is Harberger deadweight loss $H D W L_{d}^{0}$. Nearly half of $B$ is lost because the firm's most lucrative strategy is to target the high-income market, which results in the exclusion of countries like South Africa with a slightly lower income but a tremendous disease burden. But $H D W L_{d}^{0}$ understates the potential for deadweight loss from all sources, $D W L_{d}^{0}$. Given the market is not very lucrative, the manufacturer may not have an incentive to enter at all. The supremum on $D W L_{d}^{0}$ is achieved in the limit as $k_{d}$ approaches producer surplus-38\% of $B$-from above. Using the formula in Proposition 1, this supremum equals $100-38=62 \%$ of $B$.

\subsection{Goodness of Fit}

The predictions from our vaccine calibration cannot be compared to actual since an HIV vaccine is yet to be developed. We can perform the comparison for the ARTs that were developed. Our calibration, though simple, is able to match actual ART price and quantity quite closely. As discussed 
at the beginning of Section 6 , we can interpret $\$ 27,400$ as the model prediction of consumer expenditure per DALY for the HIV drug. Freedberg, et al. (2001, Table 2) estimate an actual expenditure per DALY in developed countries of $\$ 23,000$. If the $\$ 23,000$ number reflects price concessions in response to public pressure described by Reich and Bery (2005), our $\$ 27,400$ estimate could be close to the counterfactual price without public pressure.

The calibrated quantity of 1.4 million can be compared to the actual quantity of 1.3 million, computed from Figure 2 as the reported 4\% ART coverage in 2003 times the $0.5 \%$ HIV prevalence times 6.4 billion population. The predicted drug price and quantity from our calibrations both match the corresponding actual variables remarkably closely.

\subsection{Comprehensive Bound}

To obtain a comprehensive bound on deadweight loss, we move from calibrations of the vaccine and drug market in isolation to a calibration allowing for the possibility that either or both could be produced. Proposition 2 provides an exact expression for the supremum on deadweight loss in this comprehensive situation. As argued in Section 3, it simply equals the greater of the deadweightloss suprema from the isolated product markets, i.e., the greater of $56 \%$ and $62 \%$, thus equal to $62 \%$, reported in the last row of Table 4. This supremum can be approached in the limit as $k_{v}$ approaches infinity, so the vaccine market is certainly non-viable, and $k_{d}$ approaches $38 \%$ of $B$, so the drug market falls just short of the margin of viability. ${ }^{16}$

\subsection{Government Subsidies}

Kremer and Snyder (2016) note in Section 6 that Harberger deadweight loss can be highly unstable with a Zipf-similar demand curve. The monopolist may be almost indifferent between the equilibrium strategy that targets a small segment of high demand consumers and one that targets the bulk of consumers with a low price. A small subsidy may be enough to flip the equilibrium from one to the other, eliminating most of the Harberger deadweight-loss triangle.

\footnotetext{
${ }^{16}$ To see the improvement that Proposition 2 entails over previous results, compare the tight bound of $62 \%$ reported here to the bound from Proposition 15 of Kremer and Snyder (2015), equal to $\rho_{\max }^{0}-\rho_{\min }^{0}=44-38=6 \%$. The new bound point-identifies worst-case deadweight loss at $62 \%$ of $B$. The old bound tells us that the deadweightloss supremum lies somewhere in the interval between $6 \%$ and $100 \%$ of $B$, a fairly uninformative statement in this calibration.
} 
Our baseline calibrations display exactly this property. Consider the calibrated vaccine market. Less than $6 \%$ of potential consumers are served at the high equilibrium price, leading to a large Harberger triangle, amounting to $41 \%$ of $B$. Introducing a government subsidy - a per-unit subsidy, which for accounting purposes assume is paid directly to the firm - and gradually raising it in penny increments has no effect on equilibrium price or quantity until it reaches $\$ 7.69$. The next penny increment to $\$ 7.70$ causes the firm to cut the price from $\$ 130$ to zero. This relatively modest subsidy, just $6 \%$ of the pre-subsidy equilibrium price, is enough to eliminate the Harberger triangle entirely. The subsidy is socially efficient for a wide range of parameters. Even with a social cost of public funds as high as 1.8 (so raising \$1 of taxes costs society \$1.80), social welfare would be higher under the $\$ 7.70$ subsidy than in the equilibrium without it. Interestingly, what was intended to be a subsidy policy with the target of mitigating Harberger deadweight loss ends up being equivalent to a universal vaccination program.

The effect of a subsidy on the drug market is similar. Introducing a government subsidy (again, a per-unit subsidy paid directly to the firm) and gradually raising it in penny increments has no effect on equilibrium price or quantity until it reaches $\$ 941.34$. The next penny increment to $\$ 941.35$ causes the firm to cut the price from $\$ 27,387$ to $\$ 265$. While a subsidy of over $\$ 900$ might seem high, since drug prices are much higher than vaccine prices, in fact it is only $3 \%$ of the pre-subsidy equilibrium drug price. Though the subsidy does not fully eliminate the Harberger triangle, it reduces it to less than $1 \%$ of first-best social surplus as the $11 \%$ of consumers who remain unserved have very low incomes and thus very low drug demand. ${ }^{17}$ The subsidy would be socially efficient even if the social cost of public funds were as high as 2.5.

While the subsidy policy nearly eliminates Harberger deadweight loss conditional on the development of the product, it does little to eliminate the potential for deadweight loss at the extensive margin regarding whether the product is developed at all. Intuitively, the subsidies are too small to have much effect on incentives to enter the market. In the vaccine market, the $\$ 7.70$ subsidy reduces the supremum on deadweight loss by just two percentage points, from $56 \%$ to $54 \%$ of $B$. In the drug market, the $\$ 941.35$ subsidy also reduces the worst-case bound on deadweight loss by just two percentage points, from $62 \%$ to $60 \%$. Substantially improve entry incentives-thus substantially reducing deadweight loss at the extensive margin-would call for much larger subsidies.

\footnotetext{
${ }^{17}$ Eliminating the Harberger triangle entirely would require almost double the subsidy, $\$ 1,867$.
} 


\section{International Price Discrimination}

Pharmaceutical manufacturers currently have considerable ability to price discriminate across countries, but there is an active policy debate on whether this ability should be curtailed-for example, in the contexts of parallel trade for pharmaceuticals within the European Union (Danzon 1998) or re-importation of Canadian pharmaceuticals in the United States (Pecorino 2002). In contrast to the baseline calibration, which assumed the monopolist charges a uniform price across countries, the calibrations in this section allow for some form of international price discrimination, whether perfect or within limits set by reference pricing. Comparing the results across the two sections will allow us to assess the welfare effects of policies impeding price discrimination.

The results for various scenarios involving international price discrimination are presented in Table 5. For brevity, the table just presents relative producer-surplus ratios $\rho_{v}^{0}$ and $\rho_{d}^{0}$ for each scenario. The producer-surplus ratios are summary indicators of how lucrative the respective markets for a vaccine and drug are, and can easily be converted into bounds on deadweight loss using the formula in Proposition 1. The larger of the two of these gives a comprehensive bound on deadweight loss by Proposition 2. For reference, the first row of the table repeats the relative producer-surplus ratio from the baseline calibration assuming uniform pricing.

The second row of the table presents results for a calibration allowing the firm full freedom to charge different prices across countries. Since our model takes consumers to be homogeneous within a country, this pricing strategy is equivalent to perfect price discrimination, allowing the producer to extract $100 \%$ of first-best social surplus regardless of the pharmaceutical it produces. It can extract $100 \%$ of first-best social surplus from the vaccine market by selling to all individuals in country $i$ at a price of $p_{v i}^{0}=X_{i} Y_{i}^{\epsilon}$ and $100 \%$ from the drug market by selling to all infected individuals in country $i$ at a price of $p_{d i}^{0}=Y_{i}^{\epsilon}$. Thus, as reported in Table $5, \rho_{v}^{0}=\rho_{d}^{0}=100 \%$. This would eliminate any possibility of deadweight loss, whether due to inefficient pricing or entry decisions.

Governments have a variety of policies that can interfere with firms' ability to perfectly price discriminate. An international ban could shut down price discrimination entirely. A policy allowing pharmaceutical imports with no trade frictions could lead to the same result. Whether directly or indirectly implemented, a ban on price discrimination re-introduces the potential for substantial 
deadweight loss we found in the calibrations for uniform pricing. Comparing the first two rows of Table 5. In the vaccine market, the deadweight-loss supremum rises from $0 \%$ to $56 \%$ when price discrimination is banned; and in the drug market, the supremum rises from $0 \%$ to $62 \%$.

Kyle (2007) documents the range of other policies adopted by countries that impede perfect price discrimination in pharmaceutical markets. Some countries control prices directly. Others use international reference pricing, tying domestic prices to prices in peer countries. Other policies include volume rebates, profit controls, reimbursement rate adjustments, etc. Space considerations prevent us from analyzing all of these policies. We focus on just one, international reference pricing, pursued to a greater or lesser extent by nearly $80 \%$ of the countries for which Kyle (2007) could obtain dispositive evidence. We model this simply, assuming that all the countries in the world use the United States as the reference country, capping prices in their countries to be no greater than some proportion $u \in(0,1)$ of the U.S. price.

Calibrating this scenario is complicated by the endogeneity of the reference price. The firm may increase the reference price to relax the ceiling in other countries even at the sacrifice of some reference-country profits. This endogeneity is easily addressed in our simple model. There are only two relevant prices to consider charging in the United States. The firm can either decide to serve some U.S. consumers, in which case it should charge their maximum willingness to pay for product $j$ and serve all of them. If it charges a penny more, no U.S. consumers would buy. In that case, it may as well exclude the United States entirely, or equivalently set the U.S. price to infinity, allowing the firm to perfectly price discriminate across all remaining countries. We proceed by calibrating the outcome from these two strategies, one serving the United States and the other excluding it, comparing the producer surplus from each, and selecting the more lucrative one as the equilibrium.

The resulting producer-surplus ratios $\rho_{v}^{0}$ and $\rho_{d}^{0}$ are shown in Table 5 for $u$ increasing in 0.5 increments from 0 to 2 . The $u=0$ case corresponds to the case in which the United States is the sole commercial market for the good; the producer either ignores all other countries or gives the product away there. Given costless production, $c_{j}=0$, the producer is indifferent between ignoring these other countries and freely supplying them (of course consumer surplus is much higher with the latter option). Producer surplus is $37 \%$ of $B$ in this scenario whether a vaccine or drug is produced. The firm can perfectly extract the whole surplus from the U.S. market with 
either product because consumers in the country are homogeneous. This is a huge reduction in producer surplus compared to the $100 \%$ from perfect price discrimination. Yet it is not much less than the producer surplus in the uniform-pricing baseline. In other words, the firm does just about as well if it has to rely on the United States as its sole revenue source as it would if it served the whole world at a uniform price. This conclusion is particularly true in the drug market, where calibrated producer surplus ratios $\rho_{d}^{0}$ only differ by one percentage point between the uniformpricing scenario and scenario with $u=0$. One can see the basis for this result in Figure 4. In either panel, the shaded rectangle, indicating equilibrium producer surplus under uniform pricing, is not much bigger than the rectangle that could be drawn under just the slice of U.S. consumers.

Relaxing the relative-pricing constraint by increasing $u$ causes producer surplus to asymptote to the $100 \%$ maximum for perfect price discrimination, a pattern that can be seen graphically in Figure 5. Because the United States is such a high-demand country, constraining price to be half that in the U.S. $(u=0.5)$ still allows the firm to extract $80 \%$ of $B$ with a vaccine and $95 \%$ with a drug.

The last row of the table reports the producer-surplus ratio that results from excluding the United States and perfectly price discriminating among the remaining countries. This strategy allows the firm to extract $63 \%$ of $B$. That this falls well short of $100 \%$ indicates the relative importance of the U.S. market. Comparing the strategic options available to the firm, we see that the producer would prefer excluding the United States to serving only the United States. However, as long as the reference price is $u \geq 0.2$, the producer earns more from serving than excluding the United States whether it produces a vaccine or a drug.

\section{Alternative Parameterizations}

This section conducts a series of comparative-statics exercises, analyzing how the calibrations respond to alternative parameterizations. We see how the calibrations change allowing for heterogeneity in just disease risk or just income, incorporating an alternative data series for disease risk, and varying the income elasticity. The results are presented in Table 6. For brevity, again, the table just presents relative producer-surplus ratios $\rho_{v}^{0}$ and $\rho_{d}^{0}$. For reference, the first row of the table repeats the relative producer-surplus ratios from the baseline calibration. 
The first comparative-statics exercise examines what happens if countries have the same incomes, differing only in disease risk. This change makes the vaccine market much less lucrative. The top panel of Figure 6, which plots the inverse demand for this calibration, shows why. Whereas the high income and moderate HIV risk compounded to make the United States a substantial source of demand in the main calibration, the United States has now been pushed down the demand curve, removing the curve's "belly" that helped generate revenue before. Global disease risk $X_{i}$ closely follows a power law with exponent 1, the so-called Zipf distribution. Vaccine demand inherits this property, leading to an almost perfect STRZ shape. This is the worst possible shape for trying to inscribe a rectangle underneath capturing surplus for the producer. As reported in the second row of Table 6 , the vaccine producer only obtains $30 \%$ of the social surplus $B$ from completely relieving the disease burden. The firm's strategy is to serve Uganda and higher-risk countries.

The opposite picture emerges with a drug. As disease risk is resolved into disease status ex post, consumers with the same incomes have no heterogeneity. The resulting inverse demand curve for the drug, shown in the lower panel of Figure 6, is a rectangle. The drug monopolist is able to extract $100 \%$ of $B$ with a price set at consumers' homogeneous willingness to pay. There is no deadweight loss from any source, pricing or product strategy, on this market. Unfortunately, Propositions 2 and 1 tell us that the potential for deadweight loss in the comprehensive setting in which either or both products can be developed depends not on the best but on the worst outcome in the two isolated markets. Here, the comprehensive bound on relative deadweight loss equals $100-30=70 \%$. The bound can be approached in the limit as $k_{v}$ approaches $30 \%$ of $B$ and $k_{d}$ approaches infinity. More than two thirds of total surplus could be dissipated because of inadequate incentives to develop the less lucrative product, the vaccine, in this calibration.

The next comparative-statics exercise gives all countries the same HIV risk and has them just vary by income, $Y_{i}$. We omit the graph of the demand curves from now on for brevity and just look at the relative producer-surplus ratio. In this calibration, both products generate the same producer surplus. There is no change in the nature of consumer heterogeneity from the ex ante to the ex post period. The market for both products is fairly lucrative, with the producer able to capture $57 \%$ of $B$.

The next comparative-statics exercise returns to the baseline with heterogeneity in both $X_{i}$ and $Y_{i}$ but uses the revised data for 2003 HIV prevalence for $X_{i}$. Our goal is to see how robust 
our calibrations are to variation in HIV risk, which is measured with considerable error. In this alternative calibration, we use the revised data published in UNAIDS (2006) rather than the initial data published in UNAIDS (2004a). The correlation between the initial and revised HIV prevalence series is quite high, at 0.985 . There is a noticeable change in the relative producer-surplus ratios, but they remain within four percentage points of the baseline.

The next comparative-statics exercise varies the income elasticity appearing in the formulas for the willingness to pay for a vaccine $\left(X_{i} Y_{i}^{\epsilon}\right)$ and a $\operatorname{drug}\left(Y_{i}^{\epsilon}\right)$. Given that $\epsilon$ is an exogeneous rather than estimated parameter in our calibrations, and it may have important effects on demand, it is important to examine the robustness of the results to variation in $\epsilon$. Table 6 reports calibrations for 0.5 increments in $\epsilon$ from $\epsilon=0$ to $\epsilon=2$. Figure 7 shows what the pattern looks like graphically (as well as showing finer increments). The calibration for $\epsilon=0$ is a repetition of the earlier one with heterogeneity only in disease risk. The calibration for $\epsilon=1$ is a repetition of the baseline.

Looking at the overall pattern in Figure $7, \rho_{v}^{0}$ is fairly flat in $\epsilon$ over the range $\epsilon \in[0.0,0.7]$, hovering around $\rho_{v}^{0}=0.30$, falling to $\rho_{v}^{0}=0.28$ for $\epsilon=0.5$. On the other hand, $\rho_{d}^{0}$ falls precipitously over the interval $\epsilon \in[0.0,0.7]$, from $\rho_{d}^{0}=1.00$ to $\rho_{d}^{0}=0.35$. Both $\rho_{v}^{0}$ and $\rho_{d}^{0}$ turn upward for $\epsilon>0.7$ and eventually overlap each other. Intuitively, for large $\epsilon$, heterogeneity in income starts to matter more than heterogeneity in disease risk. But we know from Kremer and Snyder (2015) (see the first paragraph of Section V.B) that the two products are similarly good at generating producer surplus when there is heterogeneity in income alone. Indeed, for very large values of $\epsilon$, the very highest income country starts to dominate demand as its income is raised to an increasingly large power.

Which $\epsilon$ is empirically most plausible? We are interested in pharmaceutical sales on the private market, so in the state of nature absent government procurement or insurance coverage. Such a state is far from modern conditions at least in the United States. Getzen (2000) provides a survey of empirical studies of the income elasticity of health expenditures, locating a handful of studies using U.S. micro data from an historical period when most of the population was uninsured. Getzen finds estimates from these studies in the $[0.2,0.7]$ range. The midpoint of this interval, 0.4 , was estimated by Anderson, Collette, and Feldman (1960) using 1953 data. Micro studies using U.S. data from the modern era with more insured consumers surveyed by Getzen (2000) find income elasticities near zero, corresponding to the calibration with only disease-risk heterogeneity. Cross-country studies typically produce higher estimates of $\epsilon$. The handful of cross-country studies surveyed by 
Getzen (2000) estimated values of $\epsilon$ in the $[1.2,1.4]$ range. The 1.3 midpoint was estimated by Newhouse (1977). Figure 7 shows that relative producer surplus is higher (and the potential for deadweight loss consequently lower) at $\epsilon=1.3$ than $\epsilon=1.0$, though the estimates are fairly similar.

\section{Conclusion}

In most countries' healthcare markets, government programs overlay a complicated public or private insurance system. In this setting, it is difficult to assess how well an important market such as that for pharmaceuticals would perform in a simpler "state of nature" in which firms sold directly to consumers on a private market. This paper attempts such an assessment combining cross-country data on disease risk and income with some simple modeling assumptions to calibrate global pharmaceutical demand. As a proof of concept, we calibrate demand for an HIV vaccine and drug. Using these calibrated demands, we can compare how lucrative the products are, bound deadweight loss from pricing and entry distortions, and simulate the welfare effects of government policies.

Overall, the analysis revealed a worrisome potential for distortion in global pharmaceutical markets owing simply to the shape of pharmaceutical demand. The global demand curves for both a vaccine and a drug are Zipf similar, a shape shown in our earlier work (Kremer and Snyder 2015) to have the greatest potential for deadweight loss at both the intensive/pricing margin (i.e., the largest Harberger triangle) and the extensive/entry margin (i.e., the largest gap between private and social incentives to develop the pharmaceutical).

Our baseline calibration assumed consumers in a country share the same disease risk (equal to the prevalence of HIV in 2003, when the HIV epidemic was still growing but before antiretroviral treatments became widespread) and same income (equal to per-capital GDP) and have unit income elasticity. These simple modeling assumptions allowed us to graph the demand curves for a vaccine and a drug, inscribe the producer-surplus-maximizing rectangle underneath, derive price and quantity for the simple monopoly equilibrium we assume, and compute deadweight loss. As a reality check, we compared the predictions from the calibrations to actual in the case of HIV pharmaceuticals, ARTs, that were available circa 2003. Predictions were close to actual for price $(\$ 27,400$ versus $\$ 23,000)$ as well as quantity (1.4 million versus 1.3 million). 
In both the calibrated vaccine and drug markets, the monopolist ends up selling to a small fraction of high-demand consumers. Deadweight loss from above-cost pricing in the vaccine market is $41 \%$ of the social surplus $B$ from completely relieving the disease burden and in the drug market is $49 \%$. Potential deadweight loss from inadequate entry incentives is $56 \%$ in the vaccine market and $62 \%$ in the drug market. We proved a new proposition showing that this $62 \%$ is an exact expression for the deadweight-loss supremum in the comprehensive case in which either or both products can be developed.

We also ran a suite of calibrations allowing for price discrimination. These results highlighted the pivotal role played by the United States in global pharmaceutical demand. The firm finds targeting just the United States almost as lucrative as selling to the world at a uniform price. If other countries employ reference pricing pegged to the U.S., for most reasonable scenarios it is more lucrative for the firm to serve the United States and accept the ceiling this imposes on other prices than to exclude the United States and perfectly price discriminate across the remaining countries. The U.S. Department of Commerce (2004) has complained that OECD countries behave as free riders, relying on the high prices in relatively open U.S. markets to fund innovation while enjoying low regulatory prices themselves. Our calibrations suggest that the United States would have a pivotal role whether or not other countries regulated prices, explaining in part why other countries may be emboldened to free ride.

Our baseline analysis abstracted from the involvement of governments and insurance companies as well as epidemiological externalities. These factors can be integrated back into the analysis under a suitable reinterpretation of the model. Rather than assuming consumers are homogeneous within each country, assume national governments (or large national insurers) decide whether to order a pharmaceutical on behalf of heterogeneous citizens enrolled their health systems. Assume further that the governments only purchase pharmaceuticals satisfying the WHO's standard of high cost effectiveness, i.e., saving a DALY at a cost less than the country's GDP per capita. Assume finally that taking either pharmaceutical saves one DALY (so, for example, taking an HIV drug for a year extends the infected person's life for a year). Country $i$ 's pharmaceutical demands are the same under these assumptions as in our baseline model with direct sales to homogeneous consumers in the country with disease risk $X_{i}$, willingness to pay $Y_{i}$ given by GDP per capita, harm $H_{i}$ normalized to 1 , and income elasticity set to $\epsilon=1$. The model of national-government purchases 
can also handle epidemiological externalities recognizing that externalities mostly flow within a country and would be internalized by the government. ${ }^{18}$

In future work, we plan to derive a quantitative measure of a country's pivotalness for innovation incentives. We also plan to enrich the international calibration by accounting for the heterogeneity of consumers within each country. Kremer and Snyder (2016) calibrate global demand for an arbitrary product assuming a lognormal distribution of income within each country, using Pinkovskiy and Sala-i-Martin's (2009) estimates of the two lognormal parameters for each country. We are exploring the approach of calibrating global demand for a pharmaceutical by modeling disease risk and income as bivariate lognormal random variables and combining surveys and a variety of other data sources to estimate the five requisite parameters for each country.

\footnotetext{
${ }^{18}$ Several caveats apply to the model of national-government purchases. First, governments must be assumed to purchase at a uniform posted international price. If the firm were instead allowed to post country-specific prices, the outcome would be equivalent to the scenario with perfect price discrimination. If governments were instead allowed to bargain with the firm, this raises a new scenario not yet analyzed. It is easy to see that if parties engage in Nash bargaining, they will arrive at the social optimum. Letting $\alpha$ be the firm's bargaining share, the producer-surplus ratios in the bargaining model would be $\rho_{v}^{0}=\rho_{d}^{0}=\alpha$. Whether the deadweight-loss supremum is higher or lower in this bargaining scenario than in the uniform-posted-price baseline depends on $\alpha$ : if $\alpha=0.5$, then the deadweight-loss supremum is lower in the bargaining scenario, but the reverse is true for sufficiently low $\alpha$. A second caveat is that the national government's purchase must be tied to a commitment to universal access for all citizens-as, for example, Brazil committed to for ARTs in 1996 (Reich and Bery 2005)—rather than targeting the rich or otherwise higher demand consumers. A third caveat regards the interpretation of harm relieved by the pharmaceuticals. As noted at the beginning of Section 6 , if we interpret the $H_{i}=1$ normalization as a year's course of the drug extending life by a year, the parallel interpretation for the vaccine would involve a booster each year to maintain protection. If the vaccine is assumed to provide permanent protection, relieved harm would have to be scaled up by the discounted stream of expected DALYs saved.
} 


\section{Appendix: Proofs}

Proof of Proposition 1: Assume benchmark values of the parameters. Suppose the firm's only choice is to develop product $j$ or nothing. We will show that the supremum on relative deadweight loss is bounded by $1-\rho_{j}^{0}$ above and below, proving the two are equal and thus (6) holds.

A series of steps can be used to bound the supremum from below:

$$
\begin{aligned}
\sup _{\left\{k_{j} \geq 0\right\}}\left(\frac{D W L^{0}}{B}\right) & \geq \lim _{k_{j} \downarrow P S_{j}^{0}}\left(\frac{D W L^{0}}{B}\right) \\
& =\lim _{k_{j} \downarrow P S_{j}^{0}}\left(\frac{W^{00}}{B}\right) \\
& =\lim _{k_{j} \downarrow P S_{j}^{0}}\left[\frac{\max \left(T S_{j}^{00}-k_{j}, 0\right)}{B}\right] \\
& =\frac{T S_{j}^{00}-P S_{j}^{0}}{B} \\
& =\frac{B-P S_{j}^{0}}{B} \\
& =1-\rho_{j}^{0} .
\end{aligned}
$$

Equation (A1) follows since the limit point on the right-hand side is just one element of the closure of the larger set over which the supremum on the left-hand side is being taken. Equation (A2) holds because nothing is developed in equilibrium in the limit, implying $W^{0}=0$ and thus $D W L^{0}=$ $W^{00}-W^{0}=W^{00}$ in the limit. To see (A3), note that either product $j$ is developed, yielding social first-best surplus $T S_{j}^{00}-k_{j}$, or nothing, yielding 0 . The social optimum generates the maximum of these two social surpluses. Equation (A4) follows from evaluating the limit, (A5) follows from (2), and (A6) follows from the definition of $\rho_{j}^{0}$.

We next show the supremum is bounded above by $1-\rho_{j}^{0}$. If $W_{j}^{00}=0$, then $D W L_{j}^{0}=0 \leq 1-\rho_{j}^{0}$, and we are done. So suppose $W_{j}^{00}>0$ for the remainder of the paragraph. We have the following series of steps:

$$
\begin{aligned}
D W L^{0} & =W^{00}-W^{0} \\
& =W_{j}^{00}-W^{0} \\
& \leq W_{j}^{00}-\Pi_{j}^{0} \\
& =T S_{j}^{00}-k_{j}-\left(P S_{j}^{0}-k_{j}\right) \\
& =B-P S_{j}^{0} .
\end{aligned}
$$

Equation (A7) holds by definition. Equation (A8) holds since $W^{00}=\max \left(0, W_{j}^{00}\right)=W_{j}^{00}$ by maintained assumption that $W_{j}^{00}>0$. Equation (A9) holds since $W_{j}^{0} \geq \Pi_{j}^{0}$. Equation (A10) follows from substituting relevant definitions and (A11) substituting from (2) and canceling terms. Dividing (A7)-(A11) by $B$ yields

$$
\frac{D W L^{0}}{B} \leq 1-\rho_{j}^{0}
$$


Conditions (A6) and (A12) sandwich the supremum between $1-\rho_{j}^{0}$ above and below, yielding (6) as an exact equality. Q.E.D.

Proof of Proposition 2: Similar to the previous proof, we will show that the supremum on relative deadweight loss is bounded by $1-\rho_{\min }^{0}$ above and below, proving the two are equal and thus (7) holds. For concreteness, suppose throughout the proof that

$$
P S_{v}^{0} \leq P S_{d}^{0}
$$

Arguments establishing the bound for the reverse inequality are similar and omitted for brevity.

A series of steps can be used to bound the supremum from below:

$$
\begin{aligned}
\sup _{\left\{k_{v}, k_{d} \geq 0\right\}}\left(\frac{D W L^{0}}{B}\right) & \geq \lim _{k_{v} \downarrow P S_{v}^{0}, k_{d} \uparrow \infty}\left(\frac{D W L^{0}}{B}\right) \\
& =\lim _{k_{v} \downarrow P S_{v}^{0}}\left(\frac{W^{00}}{B}\right) \\
& =\lim _{k_{v} \downarrow P S_{v}^{0}}\left[\frac{\max \left(W_{v}^{00}, 0\right)}{B}\right] \\
& =\lim _{k_{v} \downarrow P S_{v}^{0}}\left[\frac{\max \left(T S_{v}^{00}-k_{v}, 0\right)}{B}\right] \\
& =1-\rho_{v}^{0} \\
& =1-\rho_{\min }^{0} .
\end{aligned}
$$

The arguments for (A14) and (A15) are similar to those for (A1) and (A2), respectively. Equation (A16) holds because the socially efficient product strategy cannot involve development of a drug, alone or together with a vaccine, for sufficiently large $k_{d}$. Hence $\sigma^{00} \in\{v, n\}$, implying $W^{00}=$ $\max \left(W_{v}^{00}, 0\right)$. Equation (A17) holds by definition. Equation (A18) follows from steps similar to (A3)-(A6), and (A19) holds by assumption (A13).

We next bound the supremum from above. We start by establishing the following inequality,

$$
D W L^{0} \leq B-P S_{\min }^{0}
$$

holds regardless of which value, $\sigma^{00} \in\{v, d, b, n\}$, the socially optimal product strategy takes on. First consider the trivial case in which $\sigma^{00}=n$. Then $D W L^{0}=W^{00}-W^{0}=0$ since $W^{00}=W^{0}=0$. But then (A20) trivially holds because $B-P S_{\min }^{0} \geq 0=D W L^{0}$.

Next, consider the non-trivial case in which $\sigma^{00} \in\{v, d, b\}$. We can establish the following series of steps:

$$
\begin{aligned}
D W L^{0} & =W^{00}-W^{0} \\
& =T S^{00}-k_{\sigma^{00}}-\left(\Pi^{0}+C S^{0}\right) \\
& \leq T S^{00}-k_{\sigma^{00}}-\Pi^{0} \\
& \leq T S^{00}-k_{\sigma^{00}}-\Pi_{\sigma^{00}}^{0} \\
& =T S^{00}-P S_{\sigma^{00}}^{0} . \\
& =B-P S_{\sigma^{00}}^{0} .
\end{aligned}
$$


Equations (A21) and (A22) follow from substituting the definitions of the relevant variables and (A23) from $C S^{0} \geq 0$. Equation (A24) holds because the equilibrium product strategy is the most profitable, implying $\Pi^{0} \geq \Pi_{\sigma^{00}}^{0}$. Equation (A25) follows from $\Pi_{\sigma^{00}}^{0}=P S_{\sigma^{00}}^{0}-k_{\sigma^{00}}$ and (A26) from (2).

We next show

$$
P S_{\sigma^{00}}^{0} \geq P S_{\min }^{0}
$$

for all $\sigma^{00} \in\{v, d, b\}$. If $\sigma^{00}=v$, then $P S_{\sigma^{00}}^{0}=P S_{v}^{0} \geq P S_{\min }^{0}$, implying (A27) holds. Similar arguments show (A27) holds if $\sigma^{00}=d$. Suppose $\sigma^{00}=b$. The firm can replicate producer surplus from a drug if both products have been developed by setting $p_{b v}=\infty$ and $p_{b d}=p_{d}^{0}$. Hence $P S_{b}^{0} \geq$ $P S_{d}^{0} \geq P S_{\min }^{0}$, implying (A27) holds, completing the proof that (A27) holds for all $\sigma^{00} \in\{v, d, b\}$.

We can now complete the proof. Combining (A26) and (A27), we have $D W L^{0} \leq B-P S_{\min }^{0}$ for all $\sigma^{00} \in\{v, d, b\}$. Combining this fact with the argument in the text following (A20) implies that (A20) holds for all $\sigma^{00} \in\{v, d, b, n\}$. Dividing (A20) by $B$,

$$
\frac{D W L^{0}}{B} \leq 1-\rho_{\min }^{0}
$$

for all $k_{v}, k_{d} \geq 0$, implying

$$
\sup _{\left\{k_{v}, k_{d} \geq 0\right\}}\left(\frac{D W L^{0}}{B}\right) \leq 1-\rho_{\min }^{0} .
$$

Conditions (A19) and (A29) sandwich the supremum between $1-\rho_{\min }^{0}$ above and below, yielding (7) as an exact equality. Q.E.D. 


\section{References}

Acemoglu, Daron and Joshua Linn (2004). "Market Size in Innovation: Theory and Evidence from the Pharmaceutical Industry," Quarterly Journal of Economics 119: 1049-1090.

Anderson, Odin W., Patricia Collette, and Jacob J. Feldman. (1960) Expenditure Patterns for Personal Health Services, 1953 and 1958: Nationwide Survey. New York: Health Information Foundation.

Arrow, Kenneth. (1962) "Economic Welfare and the Allocation of Resources for Inventions," in Richard Nelson, ed., The Rate and Direction of Inventive Activity. Princeton, N.J.: Princeton University Press.

Brito, Dagobert. L., Eytan Sheshinski, and Michael D. Intrilligator. (1991) "Externalities and Compulsory Vaccination," Journal of Public Economics 45: 69-90.

Budish, E., B. N. Roin, and H. Williams. (2015) "Do Firms Underinvest in Long-Term Research? Evidence from Cancer Clinical Trials," American Economic Review 105: 20442085. no. 19430.

Chen, Frederick and Flavio Toxvaerd. (2014) “The Economics of Vaccination,” Journal of Theoretical Biology 363: 105-117.

Danzon Patricia. M. (1998) "The Economics of Parallel Trade," PharmacoEconomics 13: 293304.

Danzon, Patricia M. and Jonathan. D. Ketcham. (2004) "Reference Pricing of Pharmaceuticals for Medicare: Evidence from Germany, the Netherlands, and New Zealand," Frontiers in Health Policy Research 7: 1-54.

Danzon, Patricia M., Y. Richard Wang, and Liang Wang. (2005) "The Impact of Price Regulation on the Launch Delay of New Drugs-Evidence from Twenty-Five Major Markets in the 1990s," Health Economics 14: 269-292.

European Commission (2004). The 2004 EU Industrial R\&D Investment Scoreboard (Volume II: Company Data). Technical Report EUR 21399 EN.

Finkelstein, Amy. (2004). "Static and Dynamic Effect of Health Policy: Evidence from the Vaccine Industry," Quarterly Journal of Economics 119: 527-564.

Francis, Peter J. (1997) "Dynamic Epidemiology and the Market for Vaccinations," Journal of Public Economics 63: 383-406.

Freedberg, Kenneth A., et al. (2001) "The Cost Effectiveness of Combination Antiretroviral Therapy for HIV Disease," New England Journal of Medicine 344: 824-831.

Garber, Alan M., Charles I. Jones, and Paul M. Romer. (2006) "Insurance and Incentives for Medical Innovation," Forum for Health Economics \& Policy 9: 1-27. 
Geoffard, Pierre-Yves and Tomas Philipson. (1997) "Disease Eradication: Public vs. Private Vaccination," American Economic Review 87: 222-230.

Gersovitz, Mark. (2003) "Births, Recoveries, Vaccinations, and Externalities," in R. Arnott, ed., Economics for an Imperfect World: Essays in Honor of Joseph E. Stiglitz, 469-483.

Gersovitz, Mark. and Jeffrey S. Hammer. (2004) "The Economical Control of Infectious Diseases," Economic Journal 114: 1-27.

Gersovitz, Mark. and Jeffrey S. Hammer. (2005) “Tax/Subsidy Policy Toward Vector-Borne Infectious Diseases," Journal of Public Economics 89: 647-674.

Getzen, Thomas E. (2000) "Health Care is an Individual Necessity and a National Luxury: Applying Multilevel Decision Models to the Analysis of Health Care Expenditures," Journal of Health Economics 19: 259-270.

Harberger, Arnold C. (1954) "Monopoly and Resource Allocation," American Economic Review 44: 77-92.

Hutubessy Raymond, Dan Chisholm, Tessa T. T. Edejer, and WHO-CHOICE. (2003) "Generalized Cost-Effectiveness Analysis for National-Level Priority-Setting in the Health Sector," Cost Effectiveness and Resource Allocation 1: 8.

Kremer, Michael and Christopher M. Snyder. (2003) "Why Are Drugs More Profitable Than Vaccines?" National Bureau of Economic Research working paper no. 9833.

Kremer, Michael and Christopher M. Snyder. (2013) "When Is Prevention More Profitable than Cure? The Impact of Time-Varying Consumer Heterogeneity," National Bureau of Economic Research working paper no. 18861.

Kremer, Michael and Christopher M. Snyder. (2015) "Preventives Versus Treatments," Quarterly Journal of Economics 130: 1167-1239.

Kremer, Michael and Christopher M. Snyder. (2016) "Worst-Case Bounds on R\&D and Pricing Distortions: Theory and Disturbing Conclusions if Consumer Values Follow the World Income Distribution," Harvard University working paper.

Kremer, Michael, Christopher M. Snyder, and Heidi L. Williams. (2012) "Optimal Subsidies for Prevention of Infectious Disease," Harvard University working paper.

Kyle, Margaret K. (2007) "Pharmaceutical Price Controls and Entry Strategies," Review of Economics and Statistics 89: 88-99.

Lakdawalla, Darius and Neeraj Sood. (2013) "Health Insurance as a Two-Part Pricing Contract," Journal of Public Economics 102: 1-12.

Newell, Richard G., Adam B. Jaffee, and Robert N. Stavins. (1999) “The Induced Innovation Hypothesis and Energy-Saving Technological Change," Quarterly Journal of Economics 114: 907-940. 
Newhouse, Joseph. P. (1977) "Medical Care Expenditure: A Cross-National Survey," Journal of Human Resources 12: 115-125.

Pecorino, Paul. (2002) "Should the US Allow Prescription Drug Reimports from Canada?" Journal of Health Economics 21: 699-708.

Pinkovskiy, Maxim and Xavier Sala-i-Martin. (2009) "Parametric Estimations of the World Distribution of Income,” National Bureau of Economic Research working paper no. 15433.

Reich, Michael R. and Priya Bery. (2005) "Expanding Global Access to ARVs: The Challenges of Prices and Patents," chapter 13 in K. H. Mayer and H. F. Pizer, eds., The AIDS Pandemic: Impact on Science and Society. New York: Academic Press.

Sood, Neeraj, Han de Vries, Italo Gutierrez, Darius N. Lakdawalla, and Dana P. Goldman. (2008) "The Effect of Regulation on Pharmaceutical Revenues: Experience in Nineteen Countries," Health Affairs 28: w125-w137.

Tirole, Jean. (1988) The Theory of Industrial Organization. Cambridge, Massachusetts: MIT Press.

UNAIDS. (2004a) 2004 Report on the Global AIDS Epidemic. Geneva: Joint United Nations Programme on HIV/AIDS.

UNAIDS. (2004b) Epidemiological Fact Sheets on HIV/AIDS and Sexually Transmitted Infections2004 Update. Geneva: Joint United Nations Programme on HIV/AIDS.

UNAIDS. (2006) 2006 Report on the Global AIDS Epidemic: A UNAIDS 10th Anniversary Special Edition. Geneva: Joint United Nations Programme on HIV/AIDS.

U.S. Department of Commerce. (2004) Pharmaceutical Price Controls in OECD Countries: Implications for U.S. Consumers, Pricing, Research and Development, and Innovation. Washington, D.C. 


\section{Tables and Figures}

Table 1: Toy Example Illustrating Calibration Method

\begin{tabular}{ccccccc}
\hline \hline$i$ & $N_{i}$ & $X_{i}$ & $Y_{i}$ & $N_{i} X_{i}$ & $X_{i} Y_{i}$ & $N_{i} X_{i} Y_{i}$ \\
\hline & & & & & \\
1 & 1,000 & 0.01 & 10,000 & 10 & 100 & 100,000 \\
2 & 1,500 & 0.05 & 9,000 & 75 & 400 & 675,000 \\
\hline \hline
\end{tabular}

Note: Fictitious data for a toy example of the calibration method.

Table 2: Descriptive Statistics for Cross-Country Data

\begin{tabular}{lccccc}
\hline \hline Variable & Notation & Mean & Std. dev. & Min. & Max. \\
& & & & & \\
\hline & & & & & \\
Population (million) & $N_{i}$ & 38.9 & 138.5 & 0.3 & $1,288.4$ \\
HIV prevalence & $X_{i}$ & 0.014 & 0.032 & 0.000 & 0.202 \\
GDP per capita & $Y_{i}$ & 7,653 & 12,375 & 106 & 64,670 \\
& & & & \\
\hline \hline
\end{tabular}

Notes: Entries are descriptive statistics for 2003 data for sample of 158 countries. We compute HIV prevalence by dividing "Estimated number of people living with HIV, adults and children, end 2003" from UNAIDS (2004), by population. GDP per capita from "GDP per capita (current US\$)" entry of World Bank Open Data, downloaded May 10, 2017 from http://data.worldbank.org/indicator/NY.GDP.PCAP.CD. Population from "Population, total" entry of World Bank Open Data, downloaded May 10, 2017 from http://data.worldbank.org/indicator/SP.POP.TOTL.

Table 3: Correlations in Cross-Country Data

\begin{tabular}{lrrr}
\hline \hline & \multicolumn{1}{c}{$N_{i}$} & \multicolumn{1}{c}{$X_{i}$} & \multicolumn{1}{c}{$Y_{i}$} \\
& & & \\
\hline$N_{i}$ & 1.00 & & \\
$X_{i}$ & -0.07 & 1.00 & \\
$Y_{i}$ & -0.04 & -0.19 & 1.00 \\
\hline \hline
\end{tabular}


Table 4: Baseline Calibration Results

\begin{tabular}{lcc}
\hline \hline Description & Formula & Result \\
\hline Vaccine alone & $p_{v}^{0}$ & \\
Price & $q_{v}^{0}$ & $\$ 130$ \\
Quantity & $\frac{C S_{v}^{0}}{B}=\gamma_{v}^{0}$ & 344 million \\
Consumer surplus & $\frac{P S_{v}^{0}}{B}=\rho_{v}^{0}$ & 0.16 \\
Producer surplus & $\frac{H D W L_{v}^{0}}{B}=1-\gamma_{v}^{0}-\rho_{v}^{0}$ & 0.44 \\
Harberger DWL & $\sup \left(\frac{D W L_{v}^{0}}{B}\right)=1-\rho_{v}^{0}$ & 0.41 \\
DWL supremum & $Z_{v}^{0}$ & 0.56 \\
Zipf similarity & & 0.66
\end{tabular}

Drug alone

Price

Quantity

Consumer surplus

Producer surplus

Harberger DWL

DWL supremum

Zipf similarity

$$
q_{d}^{0}
$$

1.4 million

0.13

0.38

0.62

0.74

Both products possible

DWL supremum

$$
\sup \left(\frac{D W L^{0}}{B}\right)=1-\rho_{\min }^{0}
$$

Notes: Baseline calibration in which consumers are heterogeneous in both disease risk $\left(X_{i}\right)$ and income $\left(Y_{i}\right)$, have unit income elasticity, and are willing to pay up to one year's income to avoid disease harm. All surpluses expressed as proportion of first-best social surplus (equivalently, as proportion of disease burden). The suprema are taken over $k_{v}, k_{d} \geq 0$. 
Table 5: Calibrations of Producer-Surplus Ratios for Various Price-Discrimination Scenarios

\begin{tabular}{lcc}
\hline \hline Scenario & Vaccine market $\rho_{v}^{0}$ & Drug market $\rho_{d}^{0}$ \\
& & \\
\hline & & 0.38 \\
Uniform-pricing baseline & 0.44 & 1.00 \\
Perfect price discrimination & 1.00 & \\
Price ceiling tied to varying proportion of U.S. price & & 0.37 \\
$\quad \begin{array}{l}u=0.0 \\
u=0.5\end{array}$ & 0.37 & 0.95 \\
$\quad \begin{array}{l}u=1.0 \\
u=1.5\end{array}$ & 0.80 & 1.00 \\
$u=2.0$ & 0.84 & 1.00 \\
Excluding U.S. to evade price ceiling & 0.88 & 0.91 \\
\end{tabular}

Notes: All entries are producer surplus as proportion of first-best social surplus (equivalently, as proportion of disease burden). Scenarios have the same parameters and functional form as the baseline, just changing the pricing strategy from uniform to one allowing prices to differ across countries. 
Table 6: Calibrations of Producer-Surplus Ratios for Alternative Parameterizations

\begin{tabular}{lcc}
\hline \hline Scenario & Vaccine market $\rho_{v}^{0}$ & Drug market $\rho_{d}^{0}$ \\
\hline Baseline parameterization & 0.44 & 0.38 \\
Just disease-risk heterogeneity & 0.30 & 1.00 \\
& & 0.57 \\
Just income heterogeneity & 0.57 & 0.42 \\
Revised disease-risk data & 0.48 & \\
Varying income elasticity & & 1.00 \\
$\epsilon=0.0$ & 0.30 & 0.40 \\
$\epsilon=0.5$ & 0.28 & 0.38 \\
$\epsilon=1.0$ & 0.44 & 0.59 \\
$\epsilon=1.5$ & 0.67 & 0.70 \\
$\epsilon=2.0$ & 0.69 & \\
\hline \hline
\end{tabular}

Notes: All entries are producer surplus as proportion of first-best social surplus (equivalently, as proportion of disease burden). Scenarios return to baseline assumption of uniform pricing. Scenarios return to baseline in all other dimensions as well except for the stated alternative. 
Figure 1: Demand Curves in Toy Example
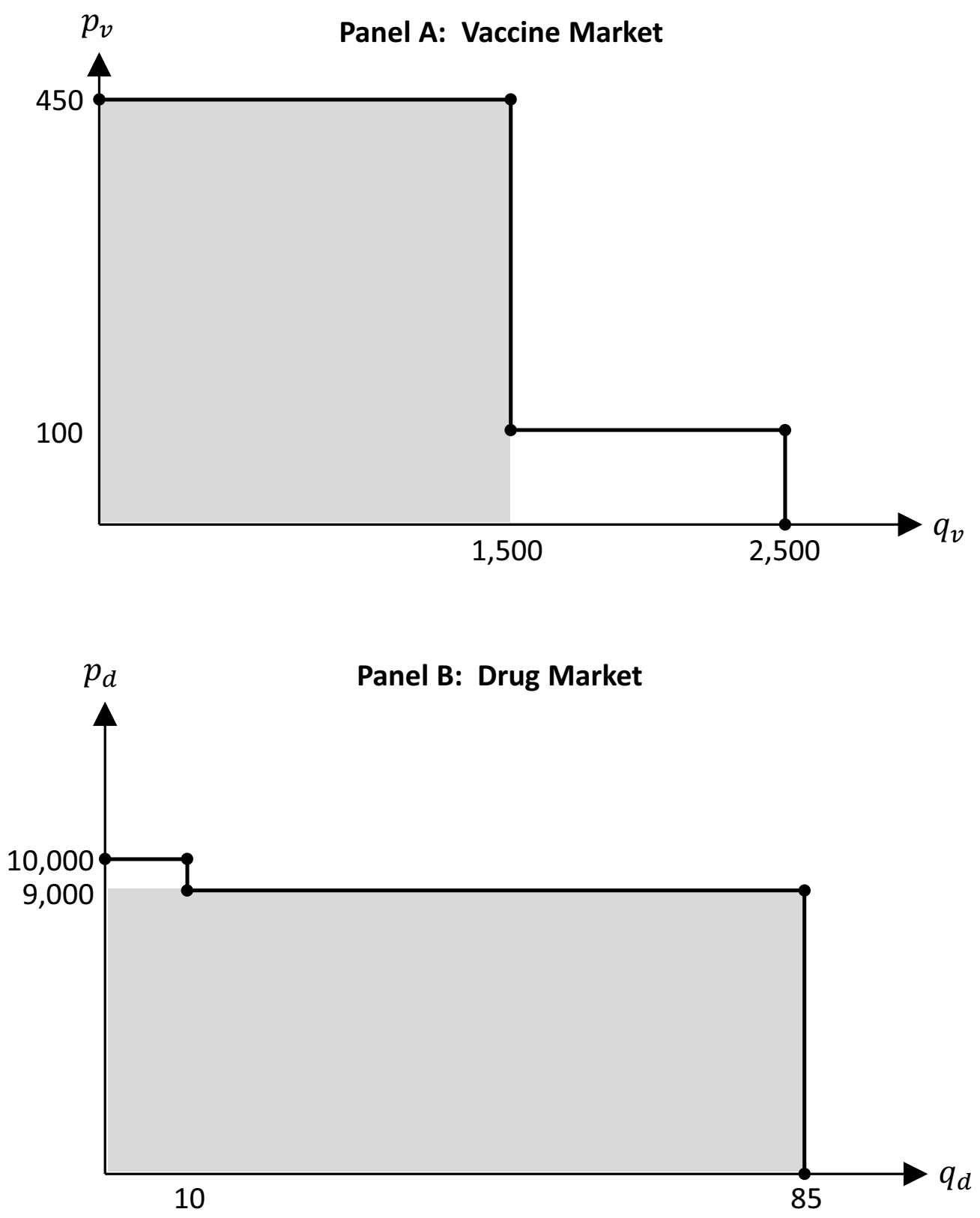

Notes: Pharmaceutical demands derived from fictitious data in Table 1. Axes scaled so that a unit of area represents the same producer surplus in both panels. Producer surplus equal to area of shaded region. 
Figure 2: Trends in Global HIV Prevalence and Treatment

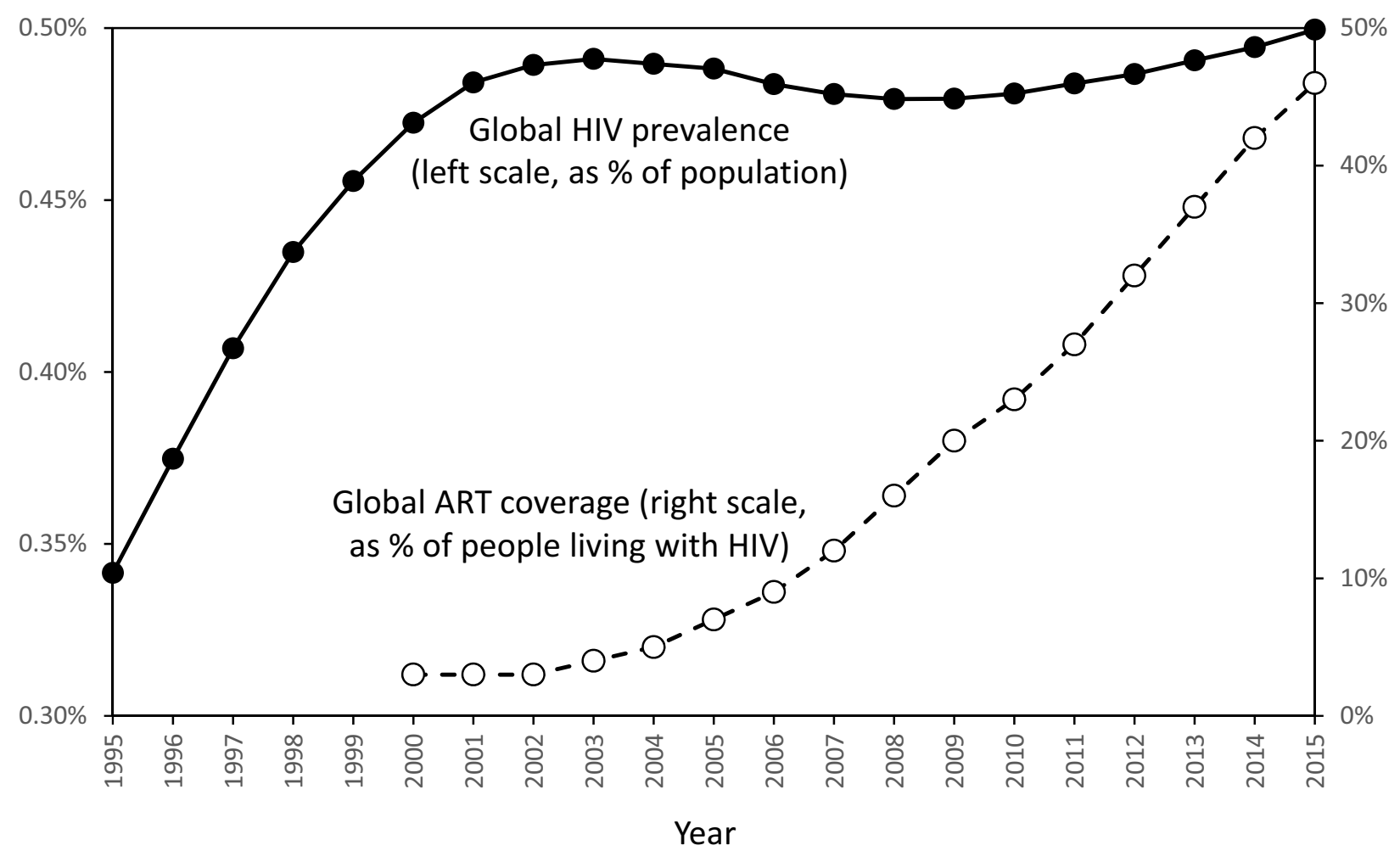

Sources: Left-axis variable equals HIV cases (from UNAIDS data on "Number of people living with HIV," downloaded from http://aidsinfo.unaids.org) as a percent of population (from World Bank data on "Population, total," downloaded from http://data.worldbank.org/indicator/SP.POP.TOTL). Right-axis variable from UNAIDS data reported by the World Bank in "Antiretroviral therapy coverage (\% of people living with HIV)," downloaded from http://data.worldbank.org/indicator/SH.HIV.ARTC.ZS. All downloads on May 8, 2017. 
Figure 3: Geographical Distribution of Main Variables
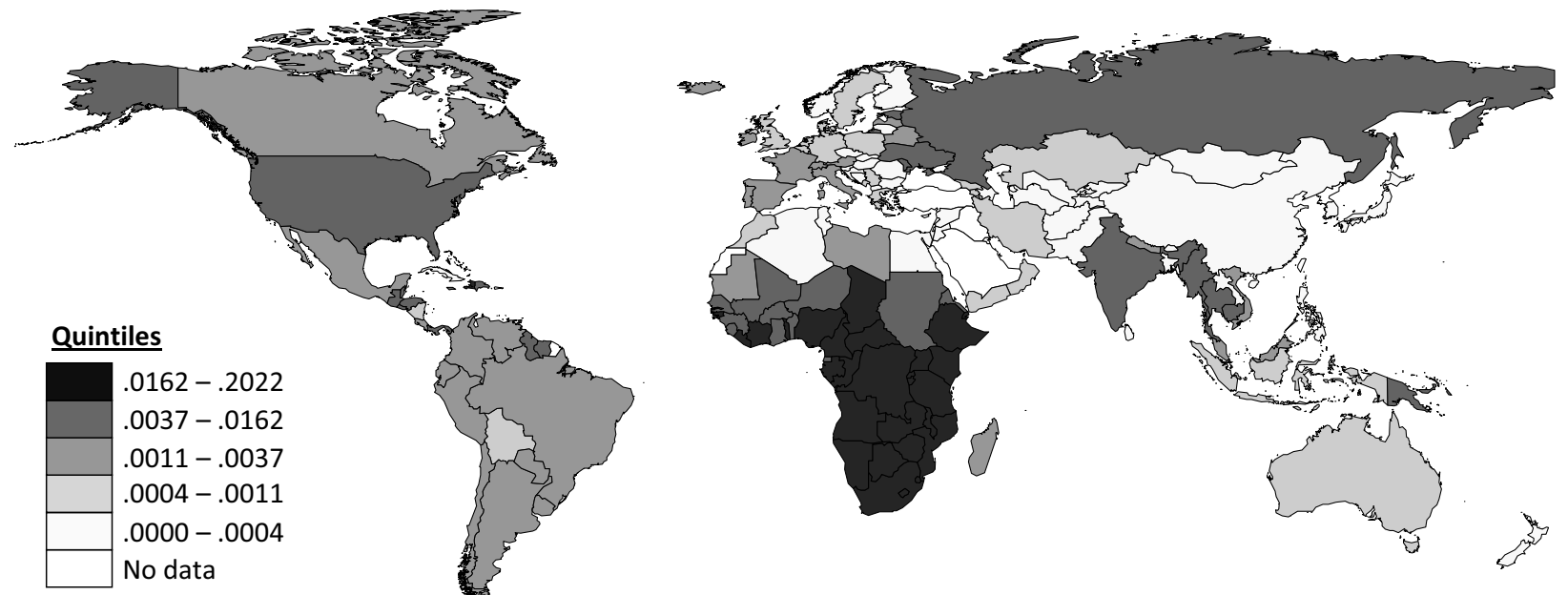

Panel A: HIV Prevalence in $2003\left(X_{i}\right)$
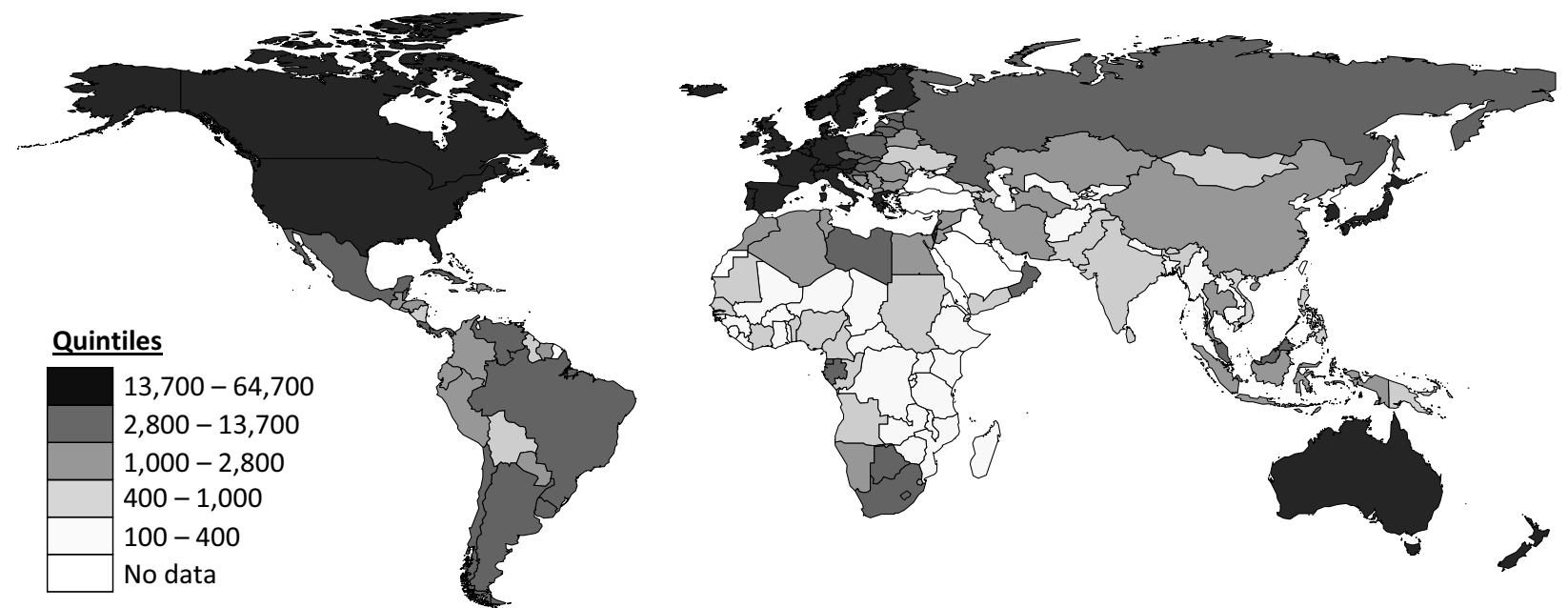

Panel B: GDP Per Capita in $2003\left(Y_{i}\right)$

Sources: See Table 2. 
Figure 4: Demand Curves for HIV Pharmaceuticals in Baseline Calibration
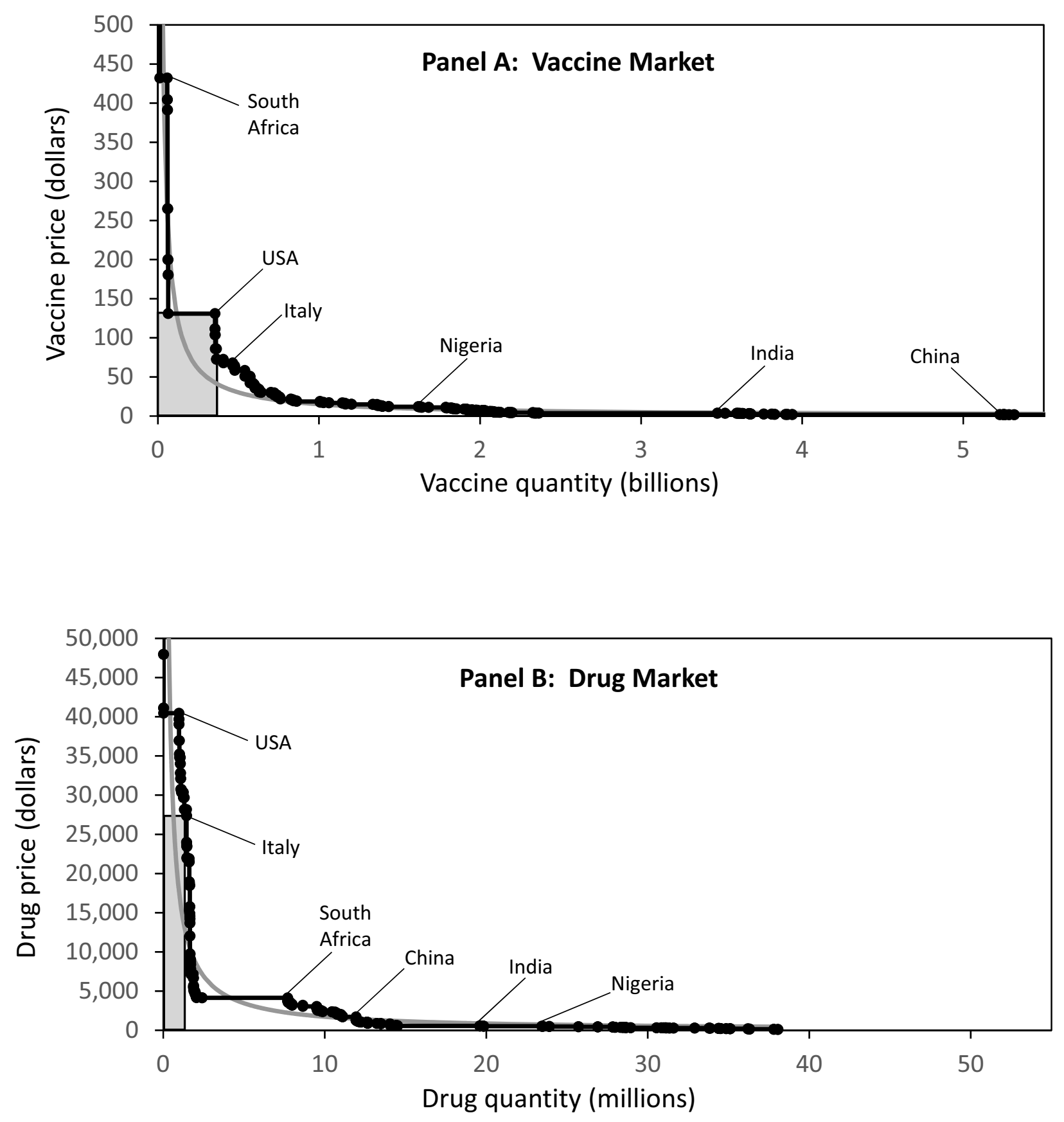

Notes: Baseline calibration, assuming heterogeneity in both disease risk $\left(X_{i}\right)$ and income $\left(Y_{i}\right)$ and income elasticity $\epsilon=1$. Axes scaled so that a unit of area represents the same producer surplus in both panels. Both axes truncated to aid visualization. Shaded rectangle represents equilibrium pricing decision; its area equals producer surplus. Grey line is STRZ demand curve with same area underneath (and thus same total surplus) as calibrated demand. 
Figure 5: Effect of Reference Pricing Pegged to the United States on Producer-Surplus Ratio

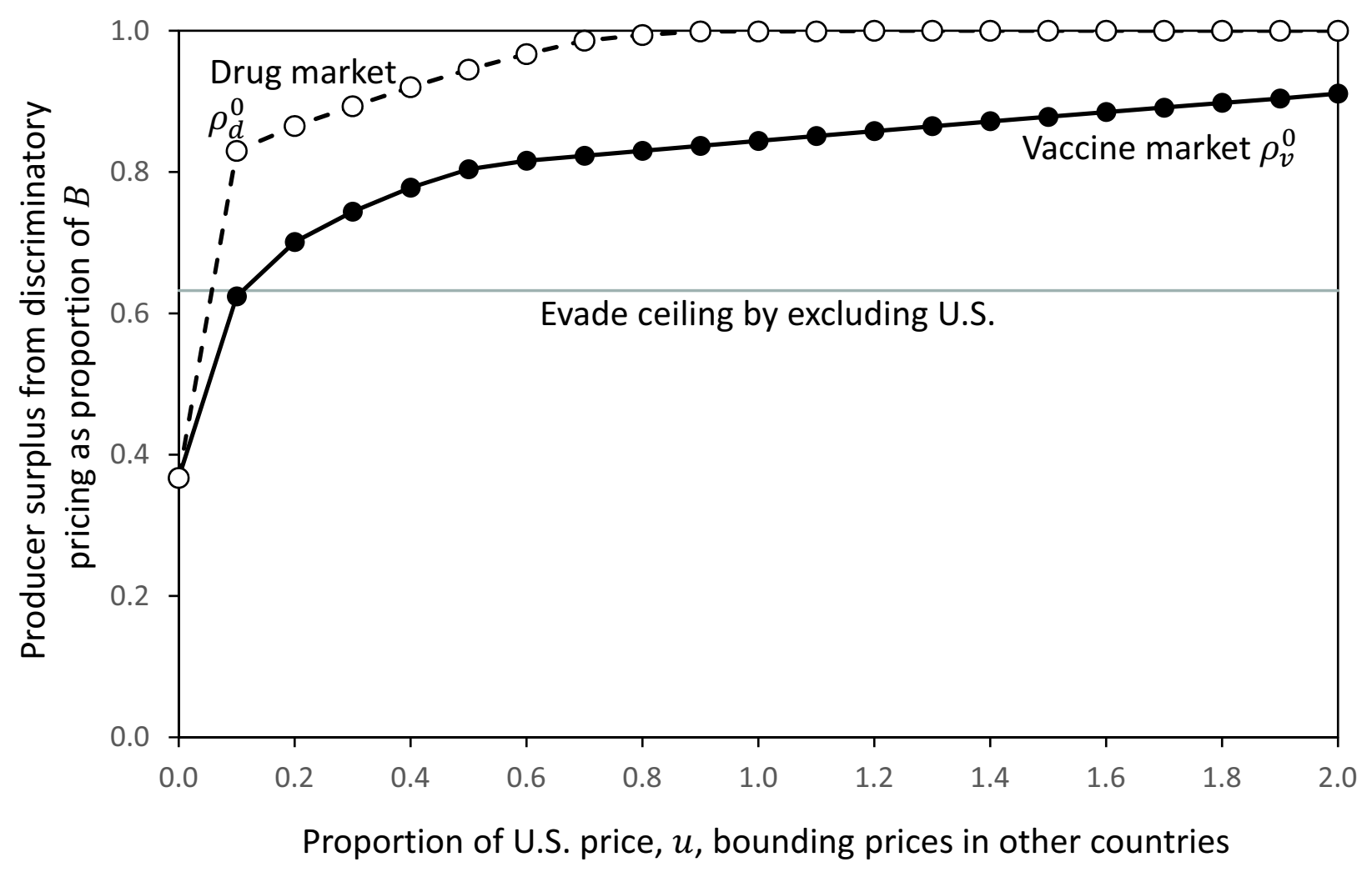


Figure 6: Demand Curves for HIV Pharmaceuticals in Calibration with Heterogeneity in Disease Risk Alone
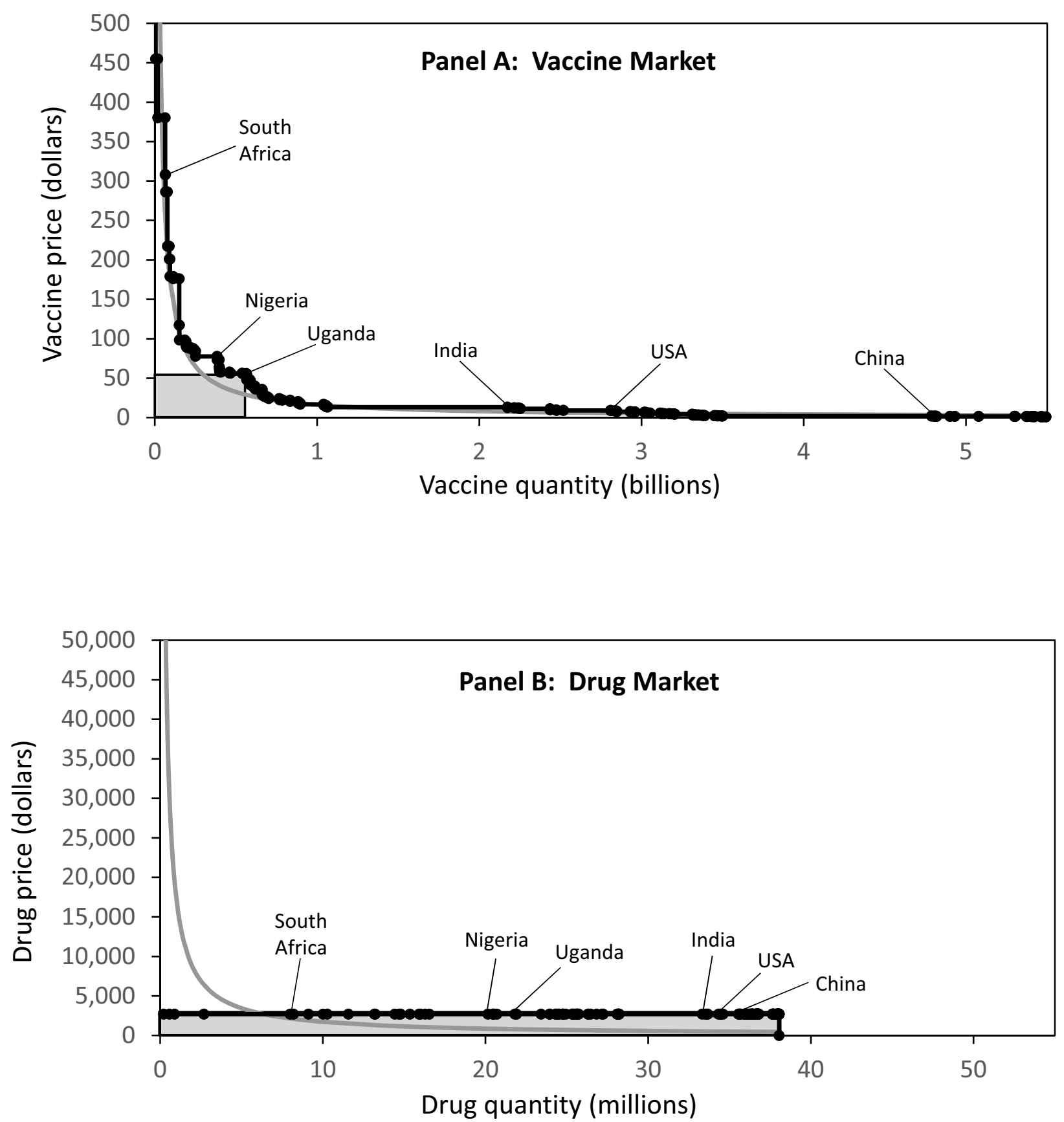

Notes: Calibration with heterogeneity in disease risk $\left(X_{i}\right)$ alone. As in previous figure, income elasticity is $\epsilon=1$. Income set to the constant $\bar{Y}=\sum_{i=1}^{I} N_{i} X_{i} Y_{i} / \sum_{i=1}^{I} N_{i} X_{i}$ such that the social surplus $B$ from completely relieving the disease burden is same as in the previous figure. One can show $\bar{Y}$ is the weighted harmonic mean of income, where country $i$ 's weight is its share of the world disease burden, $B_{i} / \sum_{k=1}^{I} B_{k}$. Axes have same scales as previous figure; as there, a unit of area represents the same producer surplus in both panels. Both axes truncated to aid visualization. Shaded rectangle represents equilibrium pricing decision; its area equals producer surplus. Grey line is STRZ demand curve with same area underneath (and thus same total surplus) as calibrated demand. 
Figure 7: Effect of Income Elasticity on Producer-Surplus Ratio

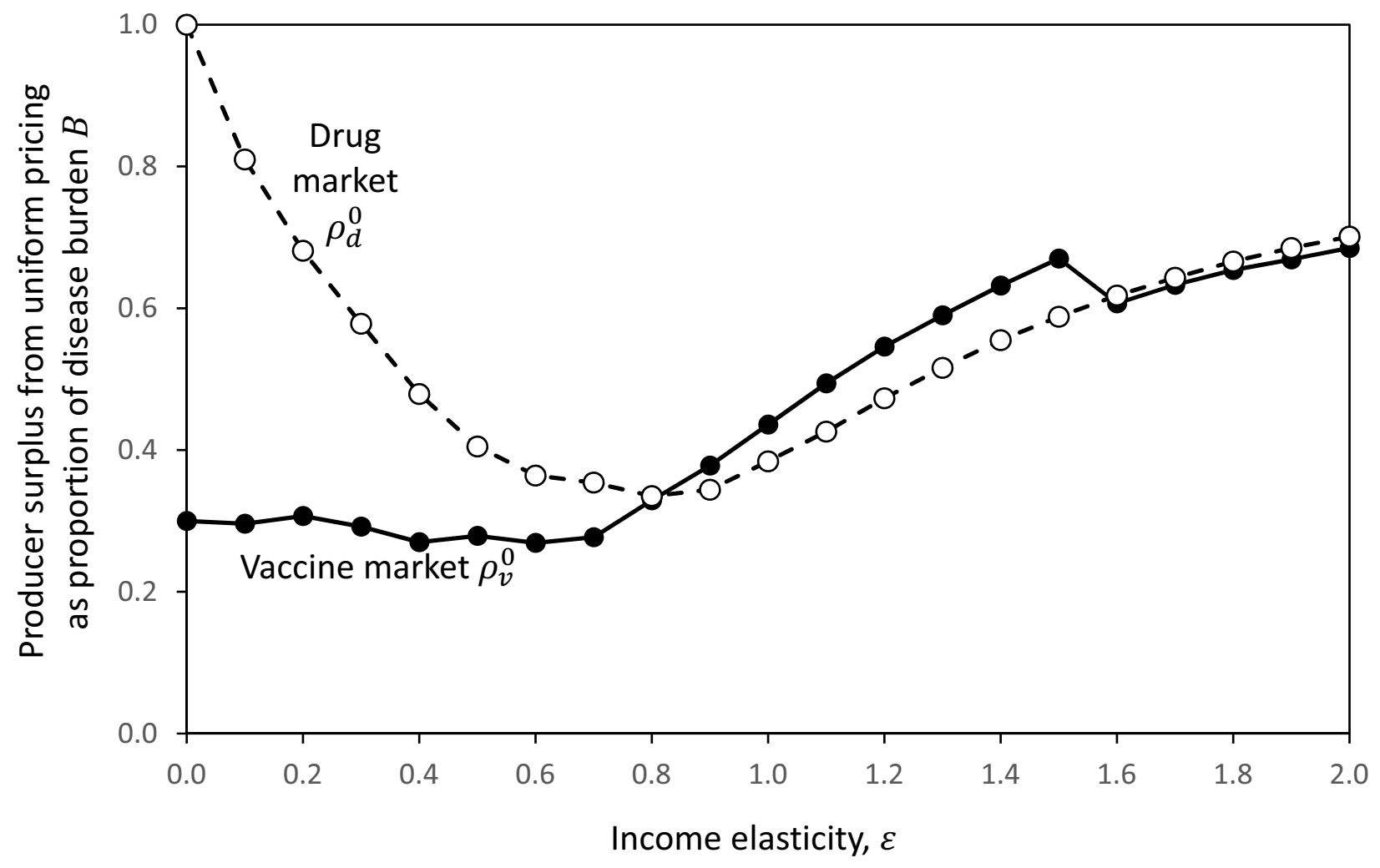

